AperTO - Archivio Istituzionale Open Access dell'Università di Torino

Pairs of nodal solutions for a class of nonlinear problems with one-sided growth conditions

This is a pre print version of the following article:

Original Citation:

Availability:

This version is available http://hdl.handle.net/2318/151279

since

Terms of use:

Open Access

Anyone can freely access the full text of works made available as "Open Access". Works made available under a Creative Commons license can be used according to the terms and conditions of said license. Use of all other works requires consent of the right holder (author or publisher) if not exempted from copyright protection by the applicable law. 


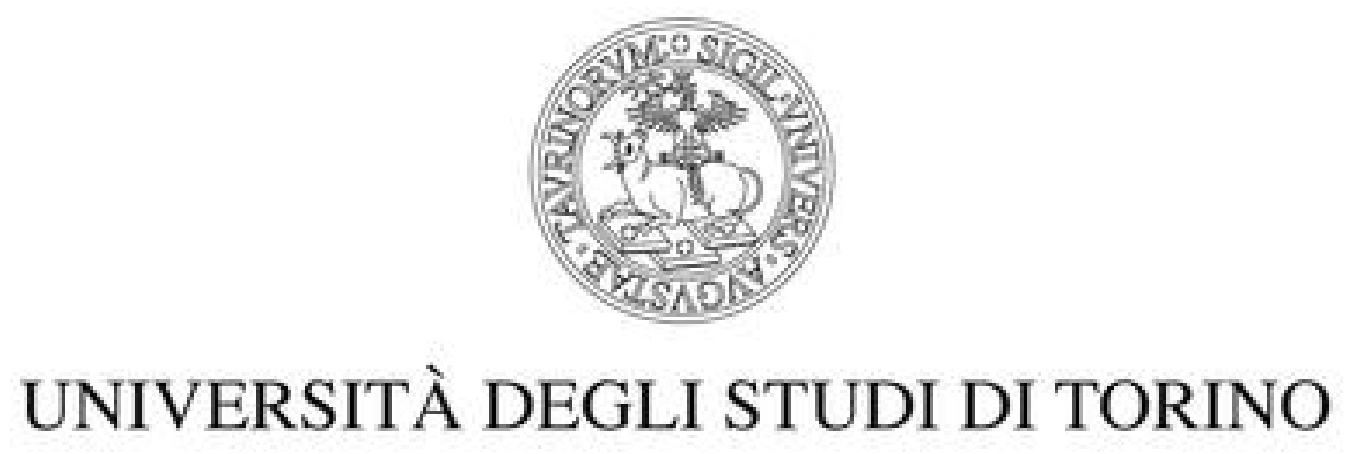

This is an author version of the contribution published on:

BOSCAGGIN A; ZANOLIN F

Pairs of nodal solutions for a class of nonlinear problems with one-sided growth conditions

ADVANCED NONLINEAR STUDIES (2013) 13 


\title{
Pairs of nodal solutions for a class of nonlinear problems with one-sided growth conditions
}

\author{
Alberto Boscaggin * \\ SISSA - ISAS, International School for Advanced Studies, \\ via Bonomea 265, 34136 Trieste - Italy \\ e-mail: boscaggi@sissa.it \\ Fabio Zanolin ${ }^{\dagger}$ \\ Department of Mathematics and Computer Science, University of Udine, \\ via delle Scienze 206, 33100 Udine - Italy \\ e-mail: fabio.zanolin@uniud.it
}

\section{Dedicated to Professor Klaus Schmitt}

\begin{abstract}
Boundary value problems of Sturm-Liouville and periodic type for the second order nonlinear ODE $u^{\prime \prime}+\lambda f(t, u)=0$ are considered. Multiplicity results are obtained for $\lambda$ positive and large under suitable growth restrictions on $f(t, u)$ of superlinear type at $u=0$ and of sublinear type at $u=\infty$. Only one-sided growth conditions are required. Applications are given to the equation $u^{\prime \prime}+\lambda q(t) \mathrm{f}(u)=0$, allowing also a weight function $q(t)$ of nonconstant sign.
\end{abstract}

Mathematics Subject Classification. 34C25, 34C28.

Key words. boundary value problems, periodic solutions, multiplicity results, rotation numbers.

${ }^{*}$ The author acknowledges the support of SISSA-ISAS, Functional Analysis Sector.

†The author acknowledges the support of the PRIN-2009 project "Ordinary Differential Equations and Applications". 


\section{Introduction}

In this paper we study the problem of existence and multiplicity of solutions to some boundary value problems associated to the second order nonlinear scalar ordinary differential equation

$$
u^{\prime \prime}+\lambda f(t, u)=0,
$$

where $f:[0, T] \times \mathbb{R} \rightarrow \mathbb{R}$ is a continuous function with $f(t, 0) \equiv 0$ and $\lambda>0$ is a real parameter.

There is a vast literature dealing with such kind of problems both for ODEs like (1.1) and for the PDE counterpart

$$
-\Delta u=\lambda f(x, u), \quad x \in \Omega,
$$

with $\Omega$ a bounded domain of $\mathbb{R}^{N}$. The special case in which $f$ splits as

$$
f(s, z)=q(s) \mathrm{f}(z)
$$

has been widely investigated for its significance in many applications and also for the interest in understanding the role of the weight function $q(s)$ with respect to existence or nonexistence results (see, for instance, [2]).

In [33], P.H. Rabinowitz proved the existence of pairs of positive solutions for the Dirichlet problem associated to (1.2) when $\lambda>0$ is large. The basic hypotheses on (1.2) require $f(x, z) / z \rightarrow 0$ for $z \rightarrow 0^{+}$uniformly in $x \in \bar{\Omega}$ and, moreover, assumptions like $f(x, z) \geq 0$ and $f \not \equiv 0$ on $\bar{\Omega} \times\left[0, \bar{z}_{0}\right]$, as well as $f(x, z) \leq 0$ on $\bar{\Omega} \times\left[\bar{z}_{1},+\infty\right)$. The hypothesis $f(x, z) \leq 0$ on large values of $z$ can be replaced by other conditions ensuring the existence of a priori bounds for the positive solutions. For instance, with this respect, one could assume $f(x, z) / z \rightarrow 0$ as $z \rightarrow+\infty$.

In some recent papers $[6,7]$ we have discussed the periodic boundary value problem for the equation

$$
u^{\prime \prime}+\lambda q(t) \mathrm{f}(u)=0
$$

in connection to Rabinowitz's results [33]. As shown in [6] the existence of positive periodic solutions is not always guaranteed and the fact that the weight function may change its sign plays a crucial role in existence/nonexistence and multiplicity results. Similar considerations apply to the Neumann problem.

One of the aims of the present paper is to continue the investigation of [6,7] with respect to the existence and multiplicity of nodal periodic solutions of (1.1), namely, periodic solutions with a prescribed number of simple zeros in $[0, T[$. The same question will be addressed to the Neumann problem.

For the Dirichlet (two-point) boundary value problem, a previous result in this direction was obtained by P.H. Rabinowitz in [34, Theorem 3] proving the existence of unbounded connected components of nodal solution $C_{k}^{ \pm} \subset \mathbb{R} \times C^{1}([0, T])$. More in detail, for every positive integer $k$, there exists $\Lambda_{k}>0$ such that for every $\lambda>\Lambda_{k}$ there exists at least two distinct points $(\lambda, u),(\lambda, v) \in C_{k}^{ \pm}$with $u(t), v(t)$ solutions of (1.1) vanishing at $t=0$ and $t=$ $T$ and having precisely $k$ zeros in $] 0, T$. Moreover $u^{\prime}(0), v^{\prime}(0)>0$ for $(\lambda, u),(\lambda, v) \in C_{k}^{+}$and $u^{\prime}(0), v^{\prime}(0)<0$ for $(\lambda, u),(\lambda, v) \in C_{k}^{-}$. Rabinowitz's theorem also applies to the equation 
$\mathcal{L} u \equiv-\left(p(t) u^{\prime}\right)^{\prime}+a(t) u=\lambda f(t, u)$ with $p(t)$ positive and continuously differentiable in $[0, T]$ and $a(t)$ non-negative and continuous. We prefer to discuss only the case $\mathcal{L} u \equiv-u^{\prime \prime}$ for uniformity in the exposition.

The assumptions on $f(s, z)$ in [34] are essentially the same as those considered in [33] for the case of positive solutions, except for the fact that now they regard two-sided conditions on $f(s, z)$, with $z \in \mathbb{R}$. Such hypotheses require a superlinear condition at zero, as

$$
\lim _{z \rightarrow 0} f(s, z) / z=0, \quad \text { uniformly in } s \in[0, T],
$$

a sign condition,

$$
f(s, z) z>0, \quad \text { for } 0<|z|<r_{0}, s \in[0, T]
$$

and an assumption at infinity given by

$$
f(s, z) z<0, \quad \text { for }|z|>r_{1}, s \in[0, T] .
$$

Concerning this latter condition, we remark that it could be replaced by other hypotheses ensuring the existence of a priori bounds for the solutions. For instance (as observed above) one could assume $f(s, z) / z \rightarrow 0$ as $|z| \rightarrow+\infty$.

Another aim of our paper is to relax the above conditions (1.5), (1.7) by assuming onesided growth restrictions on $f(s, z)$ at zero and infinity (namely only for $z>0$ or $z<0$ and for $z$ near zero or near infinity, in all the possible combinations), as well as to replace the sign condition (1.6), which is uniform with respect to $s \in[0, T]$, by a local type one. This, in particular, will permit us to obtain some new applications to (1.4) when the weight function is of nonconstant sign.

For our proofs we use a classical ODEs approach which consists in studying the qualitative properties of the trajectories in the phase-plane for the system

$$
\left\{\begin{array}{l}
x^{\prime}=-y \\
y^{\prime}=\lambda f(t, x)
\end{array}\right.
$$

and, more specifically, some planar homeomorphisms associated to the Poincare map $\Phi^{\tau}$ : $z \mapsto \zeta\left(t_{0}+\tau ; t_{0}, z\right)$ where $\zeta\left(\cdot ; t_{0}, z\right)$ is the solution of (1.8) with $\zeta\left(t_{0}\right)=z$. One of our key tools is the so-called rotation number which, roughly speaking, counts the number of turns of the solutions around the origin. Due to the special form of system (1.8), the information on the rotation number provides exact results about the nodal properties of the solutions. In this setting, the existence and multiplicity results for solutions of the Sturm-Liouville problems are obtained via a shooting type argument, while the search of the periodic solutions relies on the Poincaré-Birkhoff fixed point theorem, thanks to the Hamiltonian nature of (1.8). We notice that the Poincaré-Birkhoff theorem in its original formulation (19121913) would not apply directly to our case. Starting with Birkhoff himself [3, 4], variants of the theorem have been proposed in order to relax some hypotheses and make the result more flexible from the point of view of the applications. Some versions of the theorem are delicate, raised criticisms or have been settled only recently. For this reason and for the reader's convenience, in the first part of the Appendix we recall some basic facts about the generalized version of the Poincaré-Birkhoff theorem that we apply, in connection with the rotation number. 
A qualitative and informal presentation of our main results is depicted in Figure 1 below.

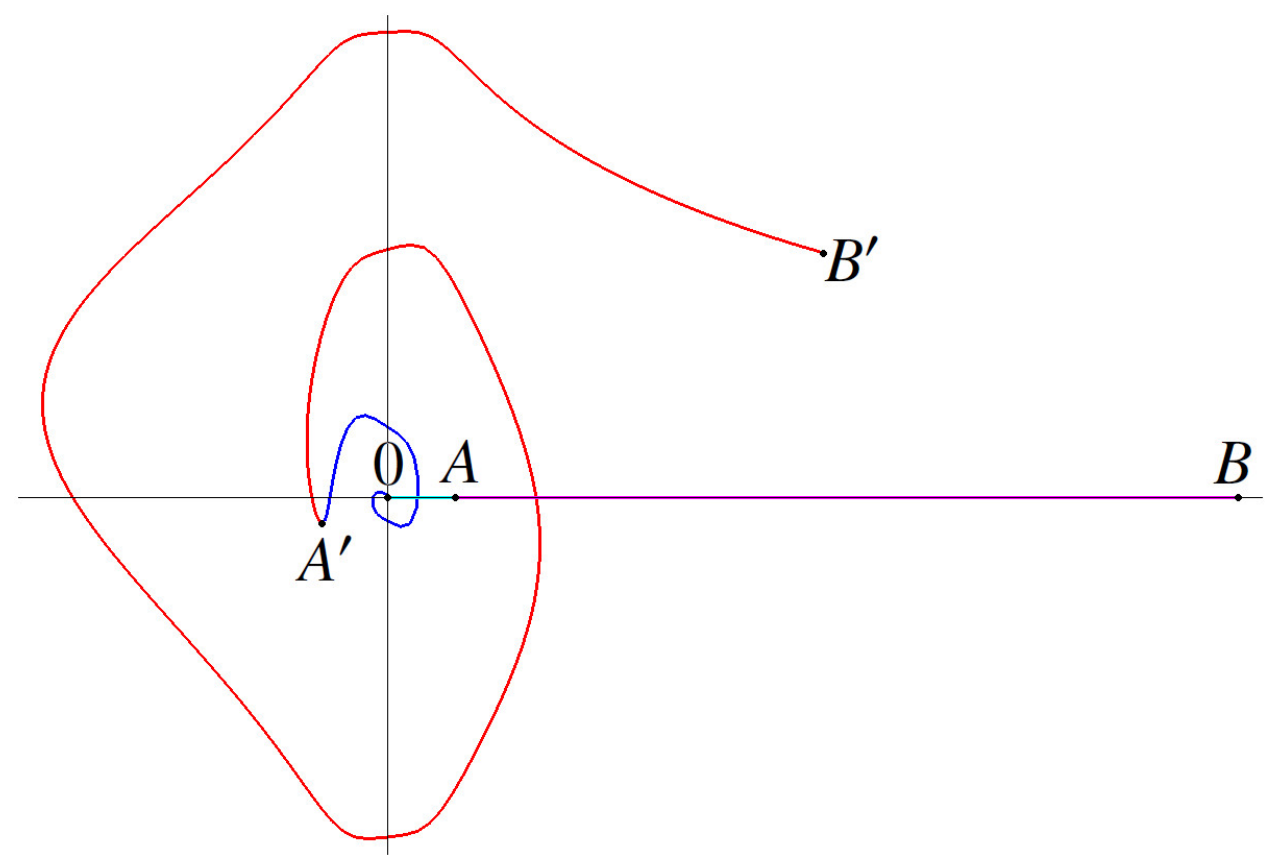

Figure 1: Example of how an half-line from the origin is deformed by the Poincaré map $\Phi$ associated to (1.8). In the present plot we have considered the image of an interval on the positive $x$-axis and taken $f(t, x)=q(t) \mathrm{f}(x)$ with $q(t) \equiv 1, f(x)=4 x^{3} /\left(1+x^{4}\right)$. For $T=1$ and $\lambda=50$ the positive $x$-axis is transformed to a double spiral winding around the origin. More precisely, the image of the segment $\overline{O A}$ through $\Phi$ is the small spiral-like arc connecting 0 to $A^{\prime}$, while the image of the segment $\overline{A B}$ is the large spiral-like arc connecting $A^{\prime}$ to $B^{\prime}$. The six nontrivial intersections of $\Phi(\overline{0 B})$ with the $x$-axis correspond to six nodal solutions of the Neumann problem starting with a positive initial value $x(0)$ with $(x(0), 0)$ on the segment $\overline{0 B}$. Indeed, we have two solutions with respectively: one zero, two zeros, three zeros in ]0,T (exactly). For each pair of such solutions, one is with a "small" initial value (namely with $(x(0), 0) \in \overline{O A}$ ) and the other is with a "large" initial value (namely with $(x(0), 0) \in \overline{A B}$ ). Other six solutions can be obtained from initial points on the negative $x$-axis. The spiral will make more winds around the origin as $\lambda$ grows, thus producing more pairs of solutions to the Neumann problem. An analogous qualitative portrait can be provided for the Dirichlet problem, by shooting from the $y$-axis. In this example the situation is particularly simple since we have considered an autonomous equation with $f$ odd. However, our theorems in Section 3 show that the same kind of result is true for a general weight function $q(t)$ (possibly of non-constant sign) and requiring on $f$ only one-sided growth conditions in zero and infinity. The periodic case is considered as well.

The plan of the paper is the following. In Section 2 we analyze the second order conservative equation $u^{\prime \prime}+\lambda f(u)=0$ by a standard phase-plane approach based on the timemappings technique (see, for instance, [11,39] for general surveys on this subject). Such an analysis, although elementary, turns out to be useful for describing some qualitative aspects of our results that will be exploited later. Section 3 is split into three parts. In the first one we state (with a few comments) our main results for equation (1.1). For simplicity, we focus our attention on three possible boundary conditions only: the Dirichlet, Neumann 
and periodic. In the second part we introduce the rotation number and prove some technical lemmas which play a crucial role in our proofs. Such lemmas deal with a first order system of the type

$$
\left\{\begin{array}{l}
x^{\prime}=-y \\
y^{\prime}=w(t, x) .
\end{array}\right.
$$

(without the parameter $\lambda$ ) and, we hope, may have some independent interest. One of our technical results requires a modified version of a classical theorem on flow-invariant sets $[1,41]$ which is presented with all the details in the second subsection of the Appendix. The last part of Section 3 is devoted to the proof of the main results. In Section 4 we show some applications and propose a few variants which can be easily derived from the main results.

Our approach, although based on classical phase-plane analysis, permits to put in evidence some peculiar dynamical aspects associated to the trajectories of (1.8). In [7] we have exploited similar properties in order to prove the presence of rich dynamics (of chaotic type) for positive solutions of equation (1.4), with $q: \mathbb{R} \rightarrow \mathbb{R}$ a continuous and $T$-periodic function, when both $\lambda$ and the negative part of $q(t)$ are large. Combining the approach in [7] with the properties of the rotation number developed in Section 3, we could provide a similar result about chaotic-like dynamics (including the existence of infinitely many subharmonics) for nodal solutions. Due to space limitations, we prefer to postpone this investigation to a future work.

The topics of the present work are related to different areas of nonlinear analysis and ordinary differential equations where Klaus Schmitt gave fundamental contributions, such as positive solutions, bifurcations and existence/multiplicity results in dependence of varying parameters, equations with a sign indefinite weight (see [19, 25, 27, 30, 40, 42], just to mention a few papers covering such topics). We are glad to dedicate our work to Professor Klaus Schmitt, with our best wishes.

\section{The autonomous case}

In this section, we focus our attention on the autonomous differential equation

$$
u^{\prime \prime}+\lambda f(u)=0,
$$

being $\lambda>0$ a parameter and $f: \mathbb{R} \rightarrow \mathbb{R}$ a continuous function satisfying the sign condition

$$
f(x) x>0, \quad \text { for every } x \neq 0 .
$$

Our analysis of the solutions of (2.1) will be performed by means of the study of the first order Hamiltonian system ${ }^{1}$

$$
x^{\prime}=-y, \quad y^{\prime}=f(x),
$$

using the simple observation that a solution $(x(t), y(t))$ of (2.3) corresponds to a solution $u(t)=x(\sqrt{\lambda} t)$ of $(2.1)$.

\footnotetext{
${ }^{1}$ Warning: we do not use the standard phase-plane setting $x^{\prime}=y, y^{\prime}=-f(x)$. Accordingly, for the solutions of (2.3) $x(t)$ is increasing/decreasing when $y(t)$ is negative/positive.
} 
We first recall that, in view of (2.2), the uniqueness for the Cauchy problems associated with (2.3) is ensured (see [35]). We also set

$$
F(x):=\int_{0}^{x} f(\xi) d \xi .
$$

By the sign condition (2.2) it clearly follows that $F(x)$ is strictly decreasing on ] $-\infty, 0]$ and strictly increasing on $[0,+\infty$ [. As well-known, each solution to (2.3) lies on a level line of the energy function

$$
\mathcal{E}(x, y):=\frac{1}{2} y^{2}+F(x), \quad(x, y) \in \mathbb{R}^{2},
$$

so that the global structure of the phase-portrait associated with (2.3) is affected by $F( \pm \infty)$.

We are now going to define some time-mapping functions associated to (2.3). For every real number $c>0$, define $] \omega^{-}(c), \omega^{+}(c)[$ as the maximal interval such that

$$
\left.F(x)<\frac{c^{2}}{2}, \quad \text { for every } x \in\right] \omega^{-}(c), \omega^{+}(c)[,
$$

and set, noticing that $\omega^{-}(c)<0<\omega^{+}(c)$,

$$
\tau^{-}(c):=\int_{\omega^{-}(c)}^{0} \frac{d \xi}{\sqrt{c^{2}-2 F(\xi)}}, \quad \tau^{+}(c):=\int_{0}^{\omega^{+}(c)} \frac{d \xi}{\sqrt{c^{2}-2 F(\xi)}} .
$$

Since the integrand is non-negative, using Lebesgue integration theory we have that $\tau^{-}(c)$ and $\tau^{+}(c)$ are well-defined, as functions with values in the set $\left.] 0,+\infty\right]$. We have the following scenario:

- if $F(+\infty)=+\infty$, then $\left.\omega^{+}(c) \mid \in\right] 0,+\infty\left[\right.$ and the map $c \mapsto \omega^{+}(c)$ is continuous; as a consequence (using standard properties of Lebesgue integrals) $\left.\tau^{+}(c) \in\right] 0,+\infty[$ and the map $c \mapsto \tau^{+}(c)$ is continuous, as well;

- if $F(+\infty)<+\infty$, we distinguish two cases:

- if $c \in] 0, \sqrt{2 F(+\infty)}\left[\right.$, then $\left.\omega^{+}(c) \in\right] 0,+\infty[$ and the same considerations as before hold true;

- if $c \geq \sqrt{2 F(+\infty)}$, then $\omega^{+}(c)=+\infty$ and $\tau^{+}(c)=+\infty$.

A symmetric situation holds with respect to $\omega^{-}(c)$ and $\tau^{-}(c)$, depending whether $F(-\infty)=$ $+\infty$ or $F(-\infty)<+\infty$.

Set

$$
c_{\infty}^{-}:=\sqrt{2 F(-\infty)}, \quad c_{\infty}^{+}:=\sqrt{2 F(+\infty)}, \quad c_{\infty}:=\min \left\{c_{\infty}^{-}, c_{\infty}^{+}\right\} .
$$

If $c<c_{\infty}^{+}$(resp., $c<c_{\infty}^{-}$), then $\tau^{+}(c)$ (resp., $\tau^{-}(c)$ ) is the time needed by a solution to (2.3) to cover, in the counterclockwise sense, the piece of orbit from $\left(\omega^{+}(c), 0\right)$ to $(0, c)$ (resp., from $(0, c)$ to $\left.\left(\omega^{-}(c), 0\right)\right)$. Hence, the origin is a local center for the solution to $(2.3)$. Indeed, every nontrivial small energy solution (small also in amplitude) is periodic, winding the origin in the counterclockwise sense. In detail, for every $c \in] 0, c_{\infty}[$ the energy level line

$$
\Gamma^{c}:=\left\{(x, y): \mathcal{E}(x, y)=c^{2} / 2\right\}
$$


is a closed orbit surrounding the origin with minimal period

$$
\tau(c):=2\left(\tau^{-}(c)+\tau^{+}(c)\right) .
$$

Notice that the center is global if and only if $c_{\infty}=+\infty$. On the other hand, if $c_{\infty}<+\infty$, some of the energy level lines are unbounded. More precisely, let us suppose that $c_{\infty}^{+}<+\infty$. In this case the solution of (2.3) departing for the point $\left(0, y_{0}\right)$ with $y_{0}<0$, meets again the $y$-axis (at the symmetric point $\left(0,\left|y_{0}\right|\right)$ if and only if $\left|y_{0}\right|<c_{\infty}^{+}$. Otherwise, if $\left|y_{0}\right| \geq c_{\infty}^{+}$, the corresponding solution $(x(t), y(t))$ of (2.3) lies on the unbounded line $y=-\sqrt{y_{0}^{2}-2 F(x)}$ in the fourth quadrant and hence $x(t)$ is a positive increasing function with $x(\infty) \geq 0$ and such that $2 F(x(\infty))=y_{0}^{2}$. Similar considerations apply when $c_{\infty}^{-}<+\infty$.

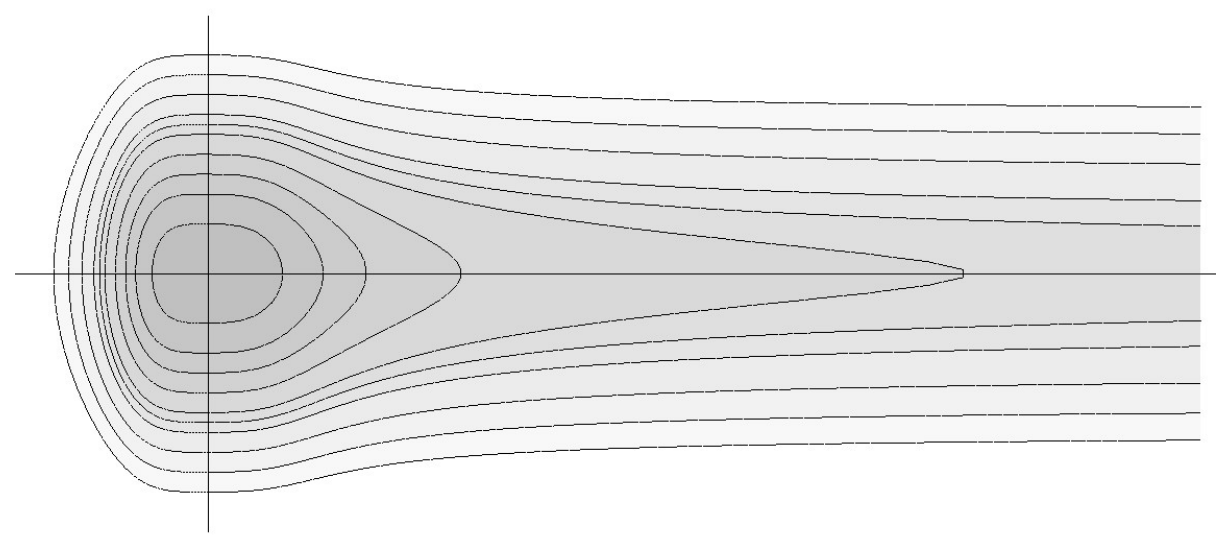

Figure 2: Example of energy level lines when $c_{\infty}^{-}=+\infty$ and $c_{\infty}^{+}<+\infty$. The function $f$ in the present example is defined as $f(x)=5 x^{3} /\left(1+x^{4}\right)$ for $x \leq 0$ and $f(x)=x^{2} /\left(1+x^{4}\right)$ for $x \geq 0$.

Our aim now is to use the time-mappings $\tau^{ \pm}$as a tool to provide multiplicity results for different boundary value problems associated to (2.1). To this end, we propose a unified approach which is independent of the finiteness of the values $c_{\infty}^{ \pm}$. From this point of view, we need to take into account both the cases in which the time-mappings are finite or are associated to an unbounded semi-orbit and thus are infinite. Hence we compactify $\mathbb{R}$ to the extended real line $\overline{\mathbb{R}}:=\mathbb{R} \cup\{-\infty,+\infty\}$ by introducing the distance

$$
d_{\overline{\mathbb{R}}}(x, y):=|\arctan (x)-\arctan (y)|,
$$

where we agree that $\arctan ( \pm \infty)= \pm \frac{\pi}{2}$. Clearly, the relative topology induced by $d_{\overline{\mathbb{R}}}$ on $\mathbb{R} \subset$ $\overline{\mathbb{R}}$ coincides with the standard topology. With these preliminaries, we have the following.

Lemma 2.1 The maps $\left.\tau^{-}, \tau^{+}: \mathbb{R}_{0}^{+}:=\right] 0,+\infty[\rightarrow \overline{\mathbb{R}}$ are continuous.

Proof. Let us fix $c_{0} \in \mathbb{R}_{0}^{+}$; we prove the continuity of $\tau^{+}$at $c_{0}$, the other case being analogous. In view of the previous discussion, the only situation to analyze is when $c_{0}=c_{\infty}^{+}=\sqrt{2 F(+\infty)}$. In this case, $\tau^{+}(c)=+\infty$ for $c \geq c_{0}$, so that the continuity in a 
right neighborhood of $c_{0}$ is ensured. The continuity in a left neighborhood follows from Fatou's lemma; indeed, since $\omega^{+}(c) \rightarrow+\infty$ for $c \rightarrow c_{0}^{-}$, we have

$$
\begin{aligned}
+\infty & =\int_{0}^{+\infty} \frac{1}{\sqrt{c_{0}^{2}-2 F(\xi)}} d \xi=\int_{0}^{+\infty}\left(\lim _{c \rightarrow c_{0}^{-}} \frac{\chi_{\left[0, \omega^{+}(c)\right]}(\xi)}{\sqrt{c^{2}-2 F(\xi)}}\right) d \xi \\
& \leq \liminf _{c \rightarrow c_{0}^{-}} \int_{0}^{+\infty} \frac{\chi_{\left[0, \omega^{+}(c)\right]}(\xi)}{\sqrt{c^{2}-2 F(\xi)}} d \xi=\liminf _{c \rightarrow c_{0}^{-}} \tau^{+}(c) .
\end{aligned}
$$

This ends the proof.

We now introduce suitable conditions for the behavior of $f(x)$ at zero and at infinity among which we are going to select the hypotheses for our theorems. Namely, we set:
$\left(f_{0}^{+}\right)$$$
\lim _{x \rightarrow 0^{+}} \frac{f(x)}{x}=0,
$$$$
\left(f_{0}^{-}\right)
$$$$
\lim _{x \rightarrow 0^{-}} \frac{f(x)}{x}=0,
$$$$
\lim _{x \rightarrow+\infty} \frac{f(x)}{x}=0
$$

$$
\lim _{x \rightarrow-\infty} \frac{f(x)}{x}=0
$$

In the sequel, we say that $f$ satisfies $\left(f_{0}\right)$ if at least one of the two conditions $\left(f_{0}^{ \pm}\right)$holds. A similar convention is applied to define $\left(f_{\infty}\right)$.

The following preliminary results are classical (see, for instance, [29]). We give the proofs for completeness.

Lemma 2.2 Assume $\left(f_{0}^{+}\right)$(resp., $\left.\left(f_{0}^{-}\right)\right)$. Then

$$
\lim _{c \rightarrow 0^{+}} \tau^{+}(c)=+\infty, \quad\left(\text { resp. }, \lim _{c \rightarrow 0^{+}} \tau^{-}(c)=+\infty\right) .
$$

Proof. Let us fix $\epsilon>0$; there exists $\delta>0$ such that $0<f(x) \leq \epsilon x$ for $x \in] 0, \delta]$. For $c$ small enough, one has $\omega^{+}(c) \leq \delta$, so that, for every $\xi \in\left[0, \omega^{+}(c)\right]$,

$$
c^{2}-2 F(\xi)=2\left(F\left(\omega^{+}(c)\right)-F(\xi)\right)=2 \int_{\xi}^{\omega^{+}(c)} f(x) d x \leq \epsilon\left(\omega^{+}(c)^{2}-\xi^{2}\right) .
$$

Hence, for every $c$ small enough,

$$
\tau^{+}(c) \geq \frac{1}{\sqrt{\epsilon}} \int_{0}^{\omega^{+}(c)} \frac{d \xi}{\sqrt{\omega^{+}(c)^{2}-\xi^{2}}}=\frac{\pi}{2 \sqrt{\epsilon}},
$$

whence the conclusion for $\epsilon \rightarrow 0^{+}$. 
Lemma 2.3 Assume $\left(f_{\infty}^{+}\right)\left(\right.$resp., $\left.\left(f_{\infty}^{-}\right)\right)$. Then

$$
\lim _{c \rightarrow\left(c_{\infty}^{+}\right)^{-}} \tau^{+}(c)=+\infty, \quad\left(\text { resp., } \quad \lim _{c \rightarrow\left(c_{\infty}^{-}\right)^{-}} \tau^{-}(c)=+\infty\right) .
$$

Proof. When $c_{\infty}^{+}<+\infty$ the conclusion follows from Lemma 2.1, since $\tau^{+}\left(c_{\infty}^{+}\right)=+\infty$. Assume now $c_{\infty}^{+}=+\infty$. Let us fix $\epsilon>0$; there exists $M>0$ such that $f(x) \leq \epsilon x$ for $x \geq M$. For $c$ large enough, one has $\omega^{+}(c) \geq 2 M$, so that, for $\xi \in\left[M, \omega^{+}(c)\right]$,

$$
c^{2}-2 F(\xi)=2\left(F\left(\omega^{+}(c)\right)-F(\xi)\right)=2 \int_{\xi}^{\omega^{+}(c)} f(x) d x \leq \epsilon\left(\omega^{+}(c)^{2}-\xi^{2}\right) .
$$

Hence, for every $c$ large enough,

$$
\tau^{+}(c) \geq \frac{1}{\sqrt{\epsilon}} \int_{M}^{\omega^{+}(c)} \frac{d \xi}{\sqrt{\omega^{+}(c)^{2}-\xi^{2}}}=\frac{1}{\sqrt{\epsilon}}\left(\frac{\pi}{2}-\arcsin \left(\frac{M}{\omega^{+}(c)}\right)\right) \geq \frac{1}{\sqrt{\epsilon}}\left(\frac{\pi}{2}-\arcsin \left(\frac{1}{2}\right)\right),
$$

whence the conclusion for $\epsilon \rightarrow+\infty$.

We are now in the position to state and prove our main results for this section. The first one deals with solutions to the Dirichlet problem.

Theorem 2.1 Assume (2.2), $\left(f_{0}\right),\left(f_{\infty}\right)$ and fix $T>0$. Then, for every positive integer $m$, there exists $\lambda_{m}^{*}>0$ such that, for every $\lambda>\lambda_{m}^{*}$, the Dirichlet problem

$$
\left\{\begin{array}{l}
u^{\prime \prime}+\lambda f(u)=0 \\
u(0)=u(T)=0
\end{array}\right.
$$

has at least $4 m$ solutions. Such solutions can be classified as follows: for every $j=$ $1, \ldots, m$, there are at least four solutions with exactly $j$ zeros in $] 0, T[;$ two of them start, respectively, with a small and with a large positive slope and the other two start with a small and with a large negative slope.

Proof. Given a positive integer $j$, let us denote by $(p(j), q(j))$ the unique pair of positive integers such that

$$
q(j) \in\{p(j)-1, p(j)\}, \quad p(j)+q(j)-1=j .
$$

Notice that

$$
q(j) \leq p(j) \leq\left\lceil\frac{j+1}{2}\right\rceil,
$$

where $\lceil\xi\rceil$ denotes the smallest integer greater or equal to the real number $\xi$.

It is then easily seen that a solution $u_{j}(t)$ to (2.4), with $j$ zeros in $] 0, T\left[\right.$ and $u_{j}^{\prime}(t)>0$, exists if and only if, for a suitable $c>0$,

$$
\frac{2}{\sqrt{\lambda}}\left(p(j) \tau^{+}(c)+q(j) \tau^{-}(c)\right)=T ;
$$


on the other hand, solutions $v_{j}(t)$ to (2.4), with $j$ zeros in $] 0, T\left[\right.$ and $v_{j}^{\prime}(t)<0$, correspond to values $c>0$ such that

$$
\frac{2}{\sqrt{\lambda}}\left(q(j) \tau^{+}(c)+p(j) \tau^{-}(c)\right)=T .
$$

At first, we fix $\left.c^{*} \in\right] 0, c_{\infty}[$; for a positive integer $m$, we define

$$
\lambda_{m}^{*}:=\left(\frac{2}{T}\left\lceil\frac{m+1}{2}\right\rceil\left(\tau^{+}\left(c^{*}\right)+\tau^{-}\left(c^{*}\right)\right)\right)^{2} .
$$

Fix now $\lambda>\lambda_{m}^{*}$ and $j \in\{1, \ldots, m\}$.

We look for solutions with positive initial slope by solving (2.6). In view of (2.5) and (2.8), it holds that

$$
\frac{2}{\sqrt{\lambda}}\left(p(j) \tau^{+}\left(c^{*}\right)+q(j) \tau^{-}\left(c^{*}\right)\right)<T
$$

on the other hand, in view of Lemma 2.2 and of Lemma 2.3, there exist $c_{1}, c_{2}>0$, with $c_{1}<c^{*}<c_{2}<c_{\infty}$, such that (according to whether $\left(f_{0}^{+}\right)$or $\left(f_{0}^{-}\right)$is satisfied)

$$
T<\frac{2}{\sqrt{\lambda}} \tau^{+}\left(c_{1}\right)<+\infty, \quad \text { or } \quad T<\frac{2}{\sqrt{\lambda}} \tau^{-}\left(c_{1}\right)<+\infty
$$

and (according to whether $\left(f_{\infty}^{+}\right)$or $\left(f_{\infty}^{-}\right)$is satisfied)

$$
T<\frac{2}{\sqrt{\lambda}} \tau^{+}\left(c_{2}\right)<+\infty \quad \text { or } \quad T<\frac{2}{\sqrt{\lambda}} \tau^{-}\left(c_{2}\right)<+\infty .
$$

Summing up, we have

$$
\frac{2}{\sqrt{\lambda}}\left(p(j) \tau^{+}\left(c^{*}\right)+q(j) \tau^{-}\left(c^{*}\right)\right)<T<\frac{2}{\sqrt{\lambda}}\left(p(j) \tau^{+}\left(c_{1}\right)+q(j) \tau^{-}\left(c_{1}\right)\right)
$$

and

$$
\frac{2}{\sqrt{\lambda}}\left(p(j) \tau^{+}\left(c^{*}\right)+q(j) \tau^{-}\left(c^{*}\right)\right)<T<\frac{2}{\sqrt{\lambda}}\left(p(j) \tau^{+}\left(c_{2}\right)+q(j) \tau^{-}\left(c_{2}\right)\right) .
$$

By Bolzano's theorem (possibly with values in $\overline{\mathbb{R}}$, since we can not exclude that $\tau^{-}(c)=+\infty$ for $c$ in a left neighborhood of $c_{2}$ ), we get the existence of $\left.c_{1}^{\#} \in\right] c_{1}, c^{*}\left[\right.$ and $\left.c_{2}^{\#} \in\right] c^{*}, c_{2}[$ satisfying (2.6), giving the two solutions to (2.4), with $j$ zeros in $] 0, T$ [ and positive initial slope, as desired.

Solutions with $j$ zeros in $] 0, T$ [ and negative initial slope are found in a completely analogous way, by looking for $\widetilde{c}_{1}, \widetilde{c}_{2}>0$, with $\widetilde{c}_{1}<c^{*}<\widetilde{c}_{2}<c_{\infty}$ satisfying (2.7).

Similarly, we have the following result for the Neumann problem. Variants for nodal solutions of other Sturm-Liouville problems could be provided, as well. 
Theorem 2.2 Assume (2.2), $\left(f_{0}\right),\left(f_{\infty}\right)$ and fix $T>0$. Then, for every positive integer $m$, there exists $\lambda_{m}^{\#}>0$ such that, for each $\lambda>\lambda_{m}^{\#}$, the Neumann problem

$$
\left\{\begin{array}{l}
u^{\prime \prime}+\lambda f(u)=0 \\
u^{\prime}(0)=u^{\prime}(T)=0,
\end{array}\right.
$$

has at least $4 m$ solutions. Such solutions can be classified as follows: for every $j=$ $1, \ldots, m$, there are at least four solutions with exactly $j$ zeros in $] 0, T[$; two of them start, respectively, with a small and with a large positive initial value and the other two start with a small and with a large negative initial value.

Finally, concerning the periodic problem, we can state the following:

Theorem 2.3 Assume $\left(f_{0}\right),\left(f_{\infty}\right)$ and fix $T>0$. Then, for every positive integer $m$, there exists $\lambda_{m}^{*}>0$ such that, for every $\lambda>\lambda_{m}^{*}$, equation (2.1) has at least $2 m$ T-periodic solutions. Precisely, for every $j=1, \ldots, m$, there are at least two $T$-periodic solutions having exactly $2 j$ zeros in $\left[0, T\left[;\right.\right.$ for one of such solutions $|u(t)|+\left|u^{\prime}(t)\right|$ is small and for the other one $|u(t)|+\left|u^{\prime}(t)\right|$ is large.

Proof. The proof follows the same arguments of those of Theorem 2.1. One has just to observe that, given a positive integer $j$, a $T$-periodic solution $u_{j}(t)$ to (2.1), with $2 j$ zeros in $[0, T[$, exists if and only if

$$
\frac{2 j}{\sqrt{\lambda}}\left(\tau^{+}(c)+\tau^{-}(c)\right)=T,
$$

for a suitable $c>0$.

Observe that, since (2.1) is an autonomous equation, every $T$-periodic solutions gives birth to a whole family of $T$-periodic solutions (all its time-translation), so that here is senseless to distinguish solutions by the sign of their initial slope (as in Theorem 2.4) or of their initial value (as in Theorem 2.10). We will recover the existence of four $T$-periodic solutions with $2 j$ zeros, for every $j=1, \ldots, m$, for the non-autonomous case, in Theorem 3.3 .

In connection with Theorems 2.2 and 2.3, notice that (since $f(x) x>0$ ) no one-signed solutions can exist. On the other hand, dealing with the Dirichlet problem, we have the following:

Theorem 2.4 Assume (2.2). Then there exists $\bar{\lambda}_{0}>0$ such that, for every $\lambda>\bar{\lambda}_{0}$, the following hold with respect to problem (2.4):

- there are at least two positive solutions (a small and a large one) when $\left(f_{0}^{+}\right)$and $\left(f_{\infty}^{+}\right)$ are satisfied;

- there are at least two negative solutions (a small and a large one) when $\left(f_{0}^{-}\right)$and $\left(f_{\infty}^{-}\right)$are satisfied;

- there are at least one positive small and one negative large solutions when $\left(f_{0}^{+}\right)$and $\left(f_{\infty}^{-}\right)$hold or one negative small and one positive large solutions when $\left(f_{0}^{-}\right)$and $\left(f_{\infty}^{+}\right)$ are satisfied. 
Proof. The proof follows the same arguments of those of Theorem 2.1. More precisely, one find a small (resp., large) positive solution by looking for a small (resp., large) value $c>0$ such that

$$
\frac{2}{\sqrt{\lambda}} \tau^{+}(c)=T
$$

and a small (resp., large) negative solution by looking for a small (resp., large) value $c>0$ such that

$$
\frac{2}{\sqrt{\lambda}} \tau^{-}(c)=T .
$$

The conclusion then follows from the Bolzano's theorem, using the preceding lemmas.

Remark 2.1 We remark that conditions $\left(f_{0}\right)$ and $\left(f_{\infty}\right)$ are just some natural assumptions which guarantee that the time-mappings tend to infinity at zero and at infinity, respectively. They are, however, not the optimal conditions and known sharper assumptions are available. For instance, according to Opial [29], the conclusion of Lemma 2.3 is still true if we assume, instead of $\left(f_{\infty}\right)$, the more general hypothesis

$$
\left(F_{\infty}\right) \quad \lim _{x \rightarrow+\infty} \frac{F(x)}{x^{2}}=0, \text { or } \lim _{x \rightarrow-\infty} \frac{F(x)}{x^{2}}=0 .
$$

Remark 2.2 The sign condition (2.2) is assumed, in this introductory section, only for the sake of simplicity. It could be replaced by the following local hypothesis:

- there exists $\delta>0$ such that

$$
f(x) x>0, \quad \text { for every } x \in[-\delta, \delta] \backslash\{0\} .
$$

In this case, we can take a maximal open interval $] x_{*}, x^{*}\left[\right.$ with $-\infty \leq x_{*}<-\delta<0<\delta<$ $x^{*} \leq+\infty$ such that $f(x) x>0$ on $] x_{*}, x^{*}\left[\backslash\{0\}\right.$. If $x^{*}<+\infty$ (resp. $x_{*}>-\infty$ ) we must have $f\left(x^{*}\right)=0$ (resp. $f\left(x_{*}\right)=0$ ). Then we modify $f$ to a new function $\widetilde{f}$ which coincides with $f(x)$ on $] x_{*}, x^{*}[$ and vanishes on $\mathbb{R} \backslash] x_{*}, x^{*}[$. The phase-plane analysis for the truncated equation $u^{\prime \prime}+\lambda \widetilde{f}(u)=0$ can be performed with some minor modifications with respect to the arguments exposed above. An elementary maximum principle reasoning allows to conclude that the solutions we find have range in $\left[x_{*}, x^{*}\right]$ and, therefore, are solutions of our original equation as well (for further details, see Corollary 4.2 in Section 4).

\section{The main results}

In this section, we state and prove our main results for the second order equation

$$
u^{\prime \prime}+\lambda f(t, u)=0,
$$

being $\lambda>0$ a parameter and $f:[0, T] \times \mathbb{R} \rightarrow \mathbb{R}$ a continuous function, with

$$
f(t, 0) \equiv 0 .
$$

Our goal is to extend the results of Section 2 to the non-autonomous equation (3.1). 


\subsection{Assumptions and statements}

At first we introduce a sign condition which generalizes (2.2). Our hypothesis is of local nature in $x$ and nonuniform in $t$. More precisely, we assume:

$\left(f_{g, h}\right)$ there exist $\delta>0$, two continuous functions $g, h:[-\delta, \delta] \rightarrow \mathbb{R}$, with

$$
0<g(x) x \leq h(x) x, \quad \text { for every } x \in[-\delta, \delta] \backslash\{0\},
$$

and a non-degenerate closed interval $I_{0}=[a, b] \subset[0, T]$ such that

$$
g(x) x \leq f(t, x) x \leq h(x) x, \quad \text { for every } t \in I_{0},|x| \leq \delta .
$$

Note that in the special case $f(t, x)=\mathrm{f}(x)$, the above condition is fulfilled if and only if $\mathrm{f}(x) x>0$ for $x \in[-\delta, \delta] \backslash\{0\}$ and we can take $g=h=\mathrm{f}$ on $[-\delta, \delta]$. If, moreover, the function $f$ takes the form

$$
f(t, x)=q(t) \mathrm{f}(x)
$$

whit $q:[0, T] \rightarrow \mathbb{R}$ continuous, then the condition $\left(f_{g, h}\right)$ is satisfied provided that $q\left(t_{0}\right)>0$ for some $t_{0} \in[0, T]$ and $\mathrm{f}(x) x>0$ for all $x \in[-\delta, \delta] \backslash\{0\}$. Indeed, in such a situation, we can choose as $[a, b]$ a suitable interval (containing $t_{0}$ ) such that $0<\min _{[a, b]} q(t)$ and take

$$
g(x):=\mathrm{f}(x) \min _{[a, b]} q(t), \quad h(x):=\mathrm{f}(x) \max _{[a, b]} q(t) .
$$

Next, we introduce some growth assumptions on $f$ at zero and at infinity which represent a natural generalization to the non-autonomous case of the conditions $\left(f_{0}\right)$ and $\left(f_{\infty}\right)$ considered above. With a little abuse in the notation we denote in the same manner also these new assumptions, so that by $\left(f_{0}\right)$ we mean that at least one of the two conditions

$\begin{array}{ll}\left(f_{0}^{+}\right) & \limsup _{x \rightarrow 0^{+}} \frac{f(t, x)}{x} \leq 0, \quad \text { uniformly in } t \in[0, T], \\ \left(f_{0}^{-}\right) & \limsup _{x \rightarrow 0^{-}} \frac{f(t, x)}{x} \leq 0, \quad \text { uniformly in } t \in[0, T],\end{array}$

holds. Similarly, by $\left(f_{\infty}\right)$ we express the fact that at least one of the two conditions

$$
\limsup _{x \rightarrow+\infty} \frac{f(t, x)}{x} \leq 0, \quad \text { uniformly in } t \in[0, T]
$$

$$
\limsup _{x \rightarrow-\infty} \frac{f(t, x)}{x} \leq 0, \quad \text { uniformly in } t \in[0, T]
$$

holds. Notice that, due to the local and nonuniform nature of the sign condition $\left(f_{g, h}\right)$, the above growth restrictions look more general than the corresponding hypotheses considered in Section 2. It is clear that, in the special case when $f(t, x)=\mathrm{f}(x)$, with $\mathrm{f}(x) x>0$ for every $x \neq 0$, the new growth conditions coincide with the previous ones. We also observe that $\left(f_{\infty}\right)$ is fulfilled whenever

$$
f(t, x) x \leq 0 \text {, for } t \in[0, T] \text { and } x \text { positive (or negative) with }|x| \text { large, }
$$

a generalization of condition (1.7) considered in Rabinowitz's theorem [34, Theorem 3]. 
To conclude with the list of the hypotheses for our main results, we add a technical condition which is required by the particular approach that we follow. Namely, we suppose that

- (*) the uniqueness and the global continuability of the solutions to the Cauchy problems associated with (3.1) are ensured.

In order to propose a few explicit assumptions which guarantee the above request for the solutions of the initial value problems, we suppose that $f(t, x)$ is locally Lipschitz continuous in $x$ and it grows at most linearly at infinity. This latter assumption can be replaced by the knowledge of some specific properties, e.g. the existence of a negative lower solution or a positive upper solution or some information about the sign of $f(t, x)$ for $|x|$ large. See Section 4 for a discussion about this topic, accompanied by the presentation of some examples. As explained in [24], there are several situations in which an explicit reference to (*) can be omitted, since we can enter in the required setting via standard tricks.

In such a framework the following results hold.

Theorem 3.1 Assume $\left(f_{g, h}\right),\left(f_{0}\right),\left(f_{\infty}\right)$. Then, for every positive integer $m$, there exists $\lambda_{m}^{*}>$ 0 such that, for each $\lambda>\lambda_{m}^{*}$, the Dirichlet problem

$$
\left\{\begin{array}{l}
u^{\prime \prime}+\lambda f(t, u)=0 \\
u(0)=u(T)=0,
\end{array}\right.
$$

has at least $4 m$ solutions. Such solutions can be classified as follows: for every $j=$ $1, \ldots, m$, there are at least four solutions with exactly $j$ zeros in $] 0, T[;$ two of them start, respectively, with a small and with a large positive slope and the other two start with a small and with a large negative slope.

Versions of Theorem 3.1 for the nodal solutions of Sturm-Liouville problems can be provided as well. For instance, for the Neumann problem, we have:

Theorem 3.2 Assume $\left(f_{g, h}\right),\left(f_{0}\right),\left(f_{\infty}\right)$. Then, for every positive integer $m$, there exists $\lambda_{m}^{\#}>$ 0 such that, for each $\lambda>\lambda_{m}^{\#}$, the Neumann problem

$$
\left\{\begin{array}{l}
u^{\prime \prime}+\lambda f(t, u)=0 \\
u^{\prime}(0)=u^{\prime}(T)=0,
\end{array}\right.
$$

has at least $4 m$ solutions. Such solutions can be classified as follows: for every $j=$ $1, \ldots, m$, there are at least four solutions with exactly $j$ zeros in $] 0, T[;$ two of them start, respectively, with a small and with a large positive initial value and the other two start with a small and with a large negative initial value.

Theorem 3.1 (as well as Theorem 3.2) is strongly related to a classical result of Rabinowitz [34, Theorem 3], which was obtained via an existence theorem in [32] providing global branches of solutions with prescribed nodal properties. For the proof of our results we use a different approach based on the evaluation of the rotation numbers associated with solution paths in the phase-plane, combined with elementary shooting arguments. Although we have preferred to focus our attention mainly on the existence and multiplicity of solutions for a fixed $\lambda$, we remark that the existence of closed connected branches of solution pairs $(\lambda, u)$ (on the line of $[32,34]$ ) could be provided too, as an application of the results developed in [38], in the frame of the shooting method. 
At the best of our knowledge, similar existence and multiplicity results for the periodic problem have not been obtained yet. Our next theorem can be seen as a contribution in this direction. As usual, by a $T$-periodic solution of (3.1) we mean a solution $u(t)$ defined on $[0, T]$ and such that

$$
u(T)-u(0)=u^{\prime}(T)-u^{\prime}(0)=0 .
$$

When $f(t, x)$ is extended by $T$-periodicity for all $t \in \mathbb{R}$, the solutions satisfying the above boundary conditions also extend to $T$-periodic solutions in the classical sense.

Theorem 3.3 Assume $\left(f_{g, h}\right),\left(f_{0}\right),\left(f_{\infty}\right)$. Then, for every positive integer $m$, there exists $\Lambda_{m}^{*}>$ 0 such that, for each $\lambda>\Lambda_{m}^{*}$, equation (3.1) has at least $4 m$ T-periodic solutions. Precisely, for every $j=1, \ldots, m$, there are at least four $T$-periodic solutions having exactly $2 j$ zeros in $\left[0, T\left[\right.\right.$; for two of such solutions $|u(t)|+\left|u^{\prime}(t)\right|$ is small and for the other two $|u(t)|+\left|u^{\prime}(t)\right|$ is large.

The terms "small" and "large" referred to the solutions in our theorems can be expressed in a form which is precisely described in the corresponding proofs. The constants $\lambda_{m}^{*}, \lambda_{m}^{\#}, \Lambda_{m}^{*}$ depend (besides, of course, on $m$ ) only on $g, h$ and the length of the interval $I_{0}$.

All the above theorems ensure the existence of solutions with a certain number of zeros in $[0, T]$. The existence of one-signed solutions is, in general, not guaranteed for the Neumann and the periodic problem without further knowledge about $f(t, x)$. With this respect, see [6] where, for the case (3.3) with $\mathrm{f}(x) x>0$ for $x \neq 0$, some conditions on the weight function $q(t)$ are found for the existence or nonexistence of positive periodic solutions. Analogous results can be derived for the Neumann problems (compare also with [2]).

On the other hand, looking for solutions of the one-dimensional Dirichlet problem, we are able to obtain existence or multiplicity results for solutions which are positive (respectively negative) in $] 0, T$ [ provided that $\left(f_{0}\right)$ and $\left(f_{\infty}\right)$ hold "on opposite sides" or "on the same side". To be more specific, the following result can be stated.

Theorem 3.4 Assume $\left(f_{g, h}\right)$. Then there exists $\bar{\lambda}_{0}>0$ such that, for every $\lambda>\bar{\lambda}_{0}$, the following hold with respect to problem (3.4):

- there are at least two positive solutions (a small and a large one) when $\left(f_{0}^{+}\right)$and $\left(f_{\infty}^{+}\right)$ are satisfied;

- there are at least two negative solutions (a small and a large one) when $\left(f_{0}^{-}\right)$and $\left(f_{\infty}^{-}\right)$are satisfied;

- there are at least one positive small and one negative large solutions when $\left(f_{0}^{+}\right)$and $\left(f_{\infty}^{-}\right)$hold, or one negative small and one positive large solutions when $\left(f_{0}^{-}\right)$and $\left(f_{\infty}^{+}\right)$ are satisfied.

The first item in Theorem 3.4 corresponds to the case considered in the classical theorem of Rabinowitz on pairs of positive solutions [33], recalled in the Introduction. In the setting of ODEs, see also [15] for a different approach based on the fixed point index for the associated operator equation in positive cones of Banach spaces. 
Dealing with the non-autonomous equation (3.1), we have assumed the continuity of the function $f$. The results could be extended in the Carathéodory setting [20], namely, for a function $f(t, x)$ which is measurable in $t$ for every $x$ and continuous in $x$ for almost every $t$, and such that for every $r>0$ there exists a $L^{\infty}$ function $\rho_{r}(t)$ such that $|f(t, x)| \leq \rho_{r}(t)$ for almost every $t \in[0, T]$ and every $x \in[-r, r]$.

\subsection{The Rotation Number: technical estimates, preliminary lemmas}

Let $\left[t_{0}, t_{1}\right] \subset \mathbb{R}$ be an interval and let $\zeta:\left[t_{0}, t_{1}\right] \rightarrow \mathbb{R}^{2} \backslash\{0\}$ be a $C^{1}$-path with $\zeta(t)=$ $(x(t), y(t))$. The rotation number of $\zeta$ counts the number of algebraic turns (in the counterclockwise sense) around the origin $0=(0,0)$ in the given time interval. It is defined as

$$
\operatorname{Rot}\left(\zeta ;\left[t_{0}, t_{1}\right]\right):=\frac{1}{2 \pi} \int_{t_{0}}^{t_{1}} \frac{x(s) y^{\prime}(s)-y(s) x^{\prime}(s)}{x(s)^{2}+y(s)^{2}} d s
$$

or, equivalently, as

$$
\operatorname{Rot}\left(\zeta ;\left[t_{0}, t_{1}\right]\right):=\frac{\theta\left(t_{1}\right)-\theta\left(t_{0}\right)}{2 \pi},
$$

once we have expressed $\zeta(t)=(x(t), y(t))$ in polar coordinates:

$$
x(t)=\rho(t) \cos \theta(t), \quad y(t)=\rho(t) \sin \theta(t),
$$

so that

$$
\theta^{\prime}(t)=\frac{1}{\rho(t)}\left(y^{\prime}(t) \cos \theta(t)-x^{\prime}(t) \sin \theta(t)\right)
$$

In some applications $\zeta(t)$ is the solution of a planar differential system

$$
\left\{\begin{array}{l}
x^{\prime}=X(t, x, y) \\
y^{\prime}=Y(t, x, y)
\end{array}\right.
$$

with $\zeta\left(t_{0}\right)=z_{0}$. In such a situation and when $\zeta(\cdot)$ is uniquely determined by $z_{0}$ as a solution of (3.7) defined on $\left[t_{0}, t_{1}\right]$, we also write $\operatorname{Rot}_{(3.7)}\left(z_{0} ;\left[t_{0}, t_{1}\right]\right)$ in place of $\operatorname{Rot}\left(\zeta ;\left[t_{0}, t_{1}\right]\right)$, so that we have

$$
\operatorname{Rot}_{(3.7)}\left(z_{0} ;\left[t_{0}, t_{1}\right]\right)=\frac{1}{2 \pi} \int_{t_{0}}^{t_{1}} \frac{x(s) Y(s, x(s), y(s))-y(s) X(s, x(s), y(s))}{x(s)^{2}+y(s)^{2}} d s .
$$

When no misunderstanding is possible, we omit to mention the reference to the differential equation and use the simplified notation $\operatorname{Rot}\left(z_{0} ;\left[t_{0}, t_{1}\right]\right)$. Note that in order to make the above formula meaningful one has to assume that the solution never vanishes. For instance, one can enter in such a situation by assuming

$$
X(t, 0,0)=Y(t, 0,0) \equiv 0 .
$$

In this case, the uniqueness of the solutions for the initial value problems ensures that $\zeta(t) \neq 0$ for all $t$, whenever $z_{0} \neq 0$.

In our approach the rotation number will be a fundamental tool to obtain multiplicity of solutions and to provide precise information about their nodal properties. To this aim we 
develop some results that, perhaps, may have some independent interest also in connection to problems not considered in the present paper. A first interesting property of the rotation numbers associated with solutions of (3.7) is expressed by the following result.

Lemma 3.1 Assume (3.8) and the uniqueness of the solutions for the Cauchy problems associated to (3.7). If

$$
X(t, 0, y) y<0, \quad \forall y \neq 0, \forall t \in\left[t_{0}, t_{1}\right],
$$

then

$$
\operatorname{Rot}_{(3.7)}\left(z_{0} ;\left[t_{0}, t_{1}\right]\right)>-1 / 2
$$

holds for every $z_{0} \neq 0$ such that the corresponding solution is defined on $\left[t_{0}, t_{1}\right]$.

Proof. The result follows by observing that $\theta^{\prime}(t)>0$ whenever $\theta(t)=\frac{\pi}{2}+j \pi$ for some $j \in \mathbb{Z}$. This makes the sets $\left\{(\rho, \theta) \in \mathbb{R}_{0}^{+} \times \mathbb{R}: \theta \geq \frac{\pi}{2}+j \pi\right\}$ positively invariant relatively to the open domain $\mathbb{R}_{0}^{+} \times \mathbb{R}$ and with respect to the differential system

$$
\left\{\begin{array}{l}
\rho^{\prime}=X(t, \rho \cos \theta, \rho \sin \theta) \cos \theta+Y(t, \rho \cos \theta, \rho \sin \theta) \sin \theta \\
\theta^{\prime}=\frac{Y(t, \rho \cos \theta, \rho \sin \theta) \cos \theta-X(t, \rho \cos \theta, \rho \sin \theta) \sin \theta}{\rho}
\end{array}\right.
$$

which comes from (3.7) passing to the polar coordinates. See [13, Lemma 2.3] or [12, Step 3] for similar considerations.

Our second result guarantees, under some weak sign conditions, that the number of turns of the solutions around the origin can become arbitrarily large provided that the considered time interval is broad enough. For our applications it is crucial that the estimates are not valid only for a single equation, but they are uniform with respect to all the vector fields which are bounded by the same comparison functions. Such a request justifies the introduction of the following notation.

- Let $\delta>0$ and let $g, h:[-\delta, \delta] \rightarrow \mathbb{R}$ be two continuous functions such that

$$
0<g(x) x \leq h(x) x, \quad \forall x \neq 0
$$

and let $I \subset \mathbb{R}$ be a compact interval. We denote by $\mathcal{W}(g, h ; I)$ the set of all continuous functions $w: \mathbb{R} \times \mathbb{R} \rightarrow \mathbb{R}$, with $w(t, 0) \equiv 0$, which satisfy the inequality

$$
g(x) x \leq w(t, x) x \leq h(x) x, \quad \text { for every } t \in I, x \in[-\delta, \delta]
$$

and such that the Cauchy problems associated with $v^{\prime \prime}+w(t, v)=0$ have a unique solution.

By the above assumptions on $w(t, x)$, it follows that for every $z_{0} \in \mathbb{R}^{2} \backslash\{0\}$, the rotation number $\operatorname{Rot}\left(z_{0} ; I\right)$ is well defined with reference to the differential system

$$
\left\{\begin{array}{l}
x^{\prime}=-y \\
y^{\prime}=w(t, x),
\end{array}\right.
$$


provided that the solution $\zeta(t)=(x(t), y(t))$ of (3.12) with value $z_{0}$ at the time $t_{0}=\min I$ is defined on the whole interval $I$.

With these positions, the following lemma holds.

Lemma 3.2 Let $g, h$ be as above. Then, for every positive integer $j$, there exist $\tau_{j}^{*}>0$ and $\left.r_{j}^{*} \in\right] 0, \delta\left[\right.$ such that, for every compact interval I with $|I|>\tau_{j}^{*}$ and for every continuous function $w \in \mathcal{W}(g, h, I)$, it holds that

$$
\operatorname{Rot}_{(3.12)}\left(z_{0} ; I\right)>j, \quad \forall z_{0} \text { with }\left|z_{0}\right|=r_{j}^{*},
$$

being $\left|z_{0}\right|$ the Euclidean norm of the point $z_{0} \in \mathbb{R}^{2}$.

Before starting the proof, we make a brief observation in order to clarify the statement of Lemma 3.2 with respect to the meaning of the property (3.13). With such a condition, we express the fact that if $z_{0}$ is any initial point with $\left|z_{0}\right|=r_{j}^{*}$ and we denote by $\zeta(t)$ the corresponding solution to (3.12) with $\zeta\left(t_{0}\right)=z_{0}$, then the rotation number on the interval $I$ is greater than $j$ if $\zeta(t)$ is defined on $I$. If $\zeta(t)$ is not defined on $I$ we consider (3.13) as vacuously satisfied. In the subsequent applications we do need to care about this fact since all the solutions will be globally defined.

Proof. The proof follows an argument previously employed in [13, 16, 21]. It consists in constructing some spiral-like curves in the phase-plane which bound from above and from below the trajectories of (3.12). With the aid of such curves one can prove that if a solution $\zeta(t)$ has a certain gap in amplitude, expressed by ||$\zeta\left(t_{1}\right)|-| \zeta\left(t_{0}\right)||$, then it must have performed a certain number of turns around the origin. Such a fact is justified by the analysis of the energy levels associated to the comparison systems

$$
x^{\prime}=-y, \quad y^{\prime}=g(x)
$$

and

$$
x^{\prime}=-y, \quad y^{\prime}=h(x),
$$

respectively. We are going to prove a local result, namely, concerning solutions in a neighborhood of the origin. For technical reasons, however, it will be convenient to suppose that $g, h$ are defined on the whole real line. Accordingly, from now on along the proof, we assume that $g, h: \mathbb{R} \rightarrow \mathbb{R}$ are continuous functions satisfying (3.10). We also introduce the potentials

$$
G(x):=\int_{0}^{x} g(\xi) d \xi, \quad H(x):=\int_{0}^{x} h(\xi) d \xi
$$

and suppose that

$$
\lim _{|x| \rightarrow+\infty} G(x)=\lim _{|x| \rightarrow+\infty} H(x)=+\infty .
$$

According to our preliminary analysis in Section 2, all the nontrivial solutions of system (3.14) and system (3.15) are periodic and lie on closed curves, which are the level lines of the energy functions

$$
\mathcal{E}_{G}(x, y):=\frac{1}{2} y^{2}+G(x), \quad \text { and } \quad \mathcal{E}_{H}(x, y):=\frac{1}{2} y^{2}+H(x)
$$


respectively.

Let $\zeta(t)=(x(t), y(t))$ be a (nontrivial) solution of (3.12) such that $|x(t)| \leq \delta$ for every $t \in\left[s_{0}, s_{1}\right] \subset I$. By a standard computation, one can see that

$$
\frac{d}{d t} \mathcal{E}_{G}(\zeta(t)) \geq 0, \text { for } x(t) y(t) \geq 0 \text { and } \frac{d}{d t} \mathcal{E}_{G}(\zeta(t)) \geq 0, \text { for } x(t) y(t) \leq 0
$$

and, symmetrically,

$$
\frac{d}{d t} \mathcal{E}_{H}(\zeta(t)) \leq 0, \text { for } x(t) y(t) \geq 0 \text { and } \frac{d}{d t} \mathcal{E}_{H}(\zeta(t)) \geq 0, \text { for } x(t) y(t) \geq 0 .
$$

As a consequence, in order to bound $\zeta(t)$ from below, we can use the level lines of $\mathcal{E}_{G}$ in the first and the third quadrant and those of $\mathcal{E}_{H}$ in the second and the fourth quadrant. Analogously, the level lines of $\mathcal{E}_{H}$ in the first and the third quadrant and those of $\mathcal{E}_{G}$ in the second and the fourth quadrant can be used to obtain upper bounds for $\zeta(t)$. Taking into account that $\zeta(t)$ winds around the origin in the counterclockwise sense, we can define two spirals departing from $\zeta\left(z_{0}\right)$ which provide a control for the solution. Such spirals are constructed by gluing level lines of $\mathcal{E}_{G}$ and $\mathcal{E}_{H}$ in alternate manner (see Figure 3 below or [16, Figure 2]).

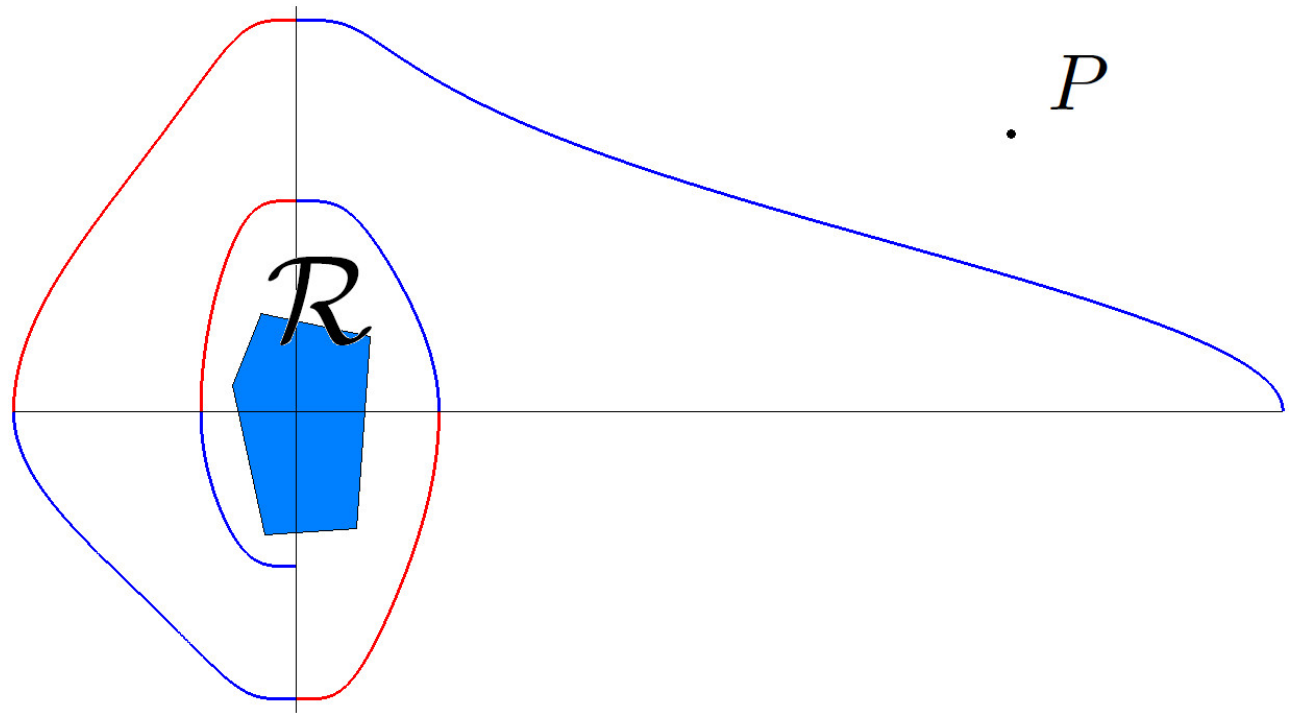

Figure 3: Example of a spiral-like curve bounding from below solutions of (3.12). For the plot we have chosen the functions $g(x)=2.8 x^{3} /\left(1+x^{4}\right)$ and $h(x)=5.2 x^{3} /\left(1+x^{4}\right)$, with $G(x)=0.7 \log \left(1+x^{4}\right)$ and $H(x)=1.3 \log \left(1+x^{4}\right)$. The spiral is obtained by alternating the level lines of $\mathcal{E}_{G}$ for $x y \geq 0$ and $\mathcal{E}_{H}$ for $x y \leq 0$. Any solution of (3.12) (for a $w \in \mathcal{W}(g, h, I)$ ) which departs from the point $P$, can enter the region $\mathcal{R}$ only after performing at least one turn and half around the origin.

Although we believe that the argument exposed above is sufficiently convincing, we prefer to present all the details in a more formal proof, by passing to the polar coordinates and using the theory of positively invariant sets. 
We introduce the auxiliary functions $\mathscr{M}=\mathscr{M}_{ \pm}(x, y): \mathbb{R}^{2} \backslash\{0\} \rightarrow \mathbb{R}$, defined as

$$
\mathscr{M}_{-}(x, y)= \begin{cases}\frac{(g(x)-x) y}{g(x) x+y^{2}} & \text { for } x y \geq 0 \\ \frac{(h(x)-x) y}{h(x) x+y^{2}} & \text { for } x y \leq 0\end{cases}
$$

and

$$
\mathscr{M}_{+}(x, y)= \begin{cases}\frac{(g(x)-x) y}{g(x) x+y^{2}} & \text { for } x y \leq 0 \\ \frac{(h(x)-x) y}{h(x) x+y^{2}} & \text { for } x y \geq 0,\end{cases}
$$

and consider the associated first order differential equations

$$
\begin{array}{ll}
\frac{d r}{d \theta}=r \mathscr{M}_{-}(r \cos \theta, r \sin \theta), & (r, \theta) \in \mathbb{R}_{0}^{+} \times \mathbb{R}, \\
\frac{d r}{d \theta}=r \mathscr{M}_{+}(r \cos \theta, r \sin \theta), & (r, \theta) \in \mathbb{R}_{0}^{+} \times \mathbb{R} .
\end{array}
$$

For every $\left(\theta_{0}, \rho_{0}\right)$ with $\theta_{0} \in \mathbb{R}$ and $\rho_{0}>0$, we denote, respectively, by $r_{-}\left(\cdot ; \theta_{0}, \rho_{0}\right)$ and $r_{+}\left(\cdot ; \theta_{0}, \rho_{0}\right)$ the solutions of (3.17) or (3.18) satisfying the initial condition $r\left(\theta_{0}\right)=\rho_{0}$.

The geometrical meaning of these solutions is the following: the maps

$$
\theta \mapsto\left(r_{ \pm}\left(\theta ; \theta_{0}, \rho_{0}\right) \cos \theta, r_{ \pm}\left(\theta ; \theta_{0}, \rho_{0}\right) \sin \theta\right)
$$

parameterize two spiralling curves, in the Cartesian plane, passing through

$$
\left(x_{0}, y_{0}\right):=\left(\rho_{0} \cos \theta_{0}, \rho_{0} \sin \theta_{0}\right)
$$

and obtained by alternating (along the motion from one quadrant to another) level curves of $\mathcal{E}_{G}$ and $\mathcal{E}_{H}$. Such spirals are precisely those we need to bound (from below and above) in the $(x, y)$-plane the solutions of (3.12).

The uniqueness of the solutions $r_{ \pm}(\theta)$ follows at once from the fundamental theory of ODEs if we assume $g, h$ continuously differentiable. One could prove the uniqueness under the sole assumption of continuity for $g, h$ thanks to the sign condition (3.10), arguing like in [35]. On the other hand, (3.10) jointly with (3.16) guarantee the global existence of the solutions. The $2 \pi$-periodicity of $\mathscr{M}_{ \pm}$in the $\theta$-variable implies

$$
r_{ \pm}\left(\theta ; \theta_{0}+2 \pi, \rho_{0}\right)=r_{ \pm}\left(\theta+2 \pi ; \theta_{0}, \rho_{0}\right), \quad \forall \theta, \theta_{0} \in \mathbb{R} \text { and } \rho_{0}>0 .
$$

At this point, we define, for every positive integer $j$ and for every $\rho_{0}>0$, the parameters

$$
m_{j}^{*}\left(\rho_{0}\right):=\inf _{\substack{\theta_{0} \in\left[0,2 \pi\left[ \\\theta \in\left[\theta_{0}, \theta_{0}+2 j \pi\right]\right.\right.}} r_{-}\left(\theta ; \theta_{0}, \rho_{0}\right), \quad M_{j}^{*}\left(\rho_{0}\right):=\sup _{\substack{\theta_{0} \in\left[0,2 \pi\left[ \\\theta \in\left[\theta_{0}, \theta_{0}+2 j \pi\right]\right.\right.}} r_{+}\left(\theta ; \theta_{0}, \rho_{0}\right) .
$$

The number $M_{j}^{*}\left(\rho_{0}\right)$ provides an upper bound for the modulus of a spiral associated to (3.18) and departing from the circumference $\rho=\rho_{0}$, while performing an angular displacement 
of $2 j \pi$. Similarly, $m_{j}^{*}\left(\rho_{0}\right)$ gives a lower bound for the modulus of the spiral associated to (3.17). For $\delta>0$ as in (3.11) and any fixed $j \geq 1$, we choose $\left.r_{j}^{*} \in\right] 0, \delta$ [ such that

$$
M_{j+1}^{*}\left(r_{j}^{*}\right)<\delta \text {. }
$$

Subsequently, we fix two numbers $\check{r}_{j}$ and $\hat{r}_{j}$ such that

$$
0<\check{r}_{j}<m_{j+1}^{*}\left(r_{j}^{*}\right) \leq r_{j}^{*} \leq M_{j+1}^{*}\left(r_{j}^{*}\right)<\hat{r}_{j}<\delta .
$$

Once we have chosen $\hat{r}_{j}$ and $\check{r}_{j}$, we can define

$$
\delta_{j}^{*}:=\inf _{\check{r}_{j} \leq \sqrt{x^{2}+y^{2}} \leq \hat{r}_{j}} \frac{g(x) x+y^{2}}{x^{2}+y^{2}}>0 .
$$

At last, we set

$$
\tau_{j}^{*}:=\frac{2 \pi j}{\delta_{j}^{*}}
$$

and, finally, we are in the position to verify that the conclusion of the Lemma holds true.

Without loss of generality, we suppose that $I=[0, \tau]$ with

$$
\tau=|I|>\tau_{j}^{*} .
$$

Let also $w \in \mathcal{W}(g, h, I)$ and let $\zeta(t)=(x(t), y(t))$ be a solution of (3.12) (which we suppose to be defined on $I$ ) with

$$
\zeta(0)=z_{0} \quad \text { and } \quad\left|z_{0}\right|=r_{j}^{*} .
$$

Passing to the polar coordinates

$$
x(t)=\rho(t) \cos \theta(t), \quad y(t)=\rho(t) \sin \theta(t), \quad \forall t \in I,
$$

we have that $(\rho(t), \theta(t))$ satisfies the differential system

$$
\left\{\begin{array}{l}
\rho^{\prime}=(w(t, \rho \cos \theta)-\rho \cos \theta) \sin \theta=: R(t, \rho, \theta) \\
\theta^{\prime}=\sin ^{2} \theta+\frac{w(t, \rho \cos \theta) \cos \theta}{\rho}=: \Theta(t, \rho, \theta) .
\end{array}\right.
$$

Moreover, $\rho(0)=r_{j}^{*}$ and, without loss of generality,

$$
\theta(0)=\theta_{0} \in[0,2 \pi[.
$$

With these positions, (3.13) turns out to be equivalent to

$$
\theta(\tau)-\theta(0)>2 \pi j
$$

For our proof the hypothesis (3.11) plays a crucial role as it permits to control from above and from below the behavior of $\zeta(t)$ in terms of the spiral-like curves previously introduced. Since (3.11) holds only locally, that is for $x \in[-\delta, \delta]$, we first produce an estimate on the angular displacement as long as the solution remains in the strip $|x| \leq \delta$. To this aim, we define as $\sigma \in] 0, \tau]$ as the maximal number such that

$$
\check{r}_{j} \leq \rho(t)=|\zeta(t)| \leq \hat{r}_{j}, \quad \forall t \in[0, \sigma] .
$$

By the choice of $\hat{r}_{j}$ we have also that $|x(t)|<\delta$ for all $t \in[0, \sigma]$ and therefore we can take advantage of the assumption (3.11) as long as $t \in[0, \sigma]$. 
We now argue differently according to the fact that $\sigma=\tau$ or $\sigma<\tau$.

- Suppose that $\sigma=\tau$. In this case, from the rotation number formula, we obtain that

$$
\begin{aligned}
\operatorname{Rot}_{(3.12)}\left(z_{0} ; I\right) & =\operatorname{Rot}(\zeta ;[0, \tau])=\frac{1}{2 \pi} \int_{0}^{\tau} \frac{y(t)^{2}+x(t) w(t, x(t))}{x(t)^{2}+y(t)^{2}} d t \\
& \geq \frac{1}{2 \pi} \int_{0}^{\tau} \frac{y(t)^{2}+x(t) g(x(t))}{x(t)^{2}+y(t)^{2}} d t \geq \delta_{j}^{*} \frac{\tau}{2 \pi}>\delta_{j}^{*} \frac{\tau_{j}^{*}}{2 \pi}=j
\end{aligned}
$$

and hence the thesis follows.

- Assume that $\sigma<\tau$. In this case, the maximality of $\sigma$ implies that

$$
\check{r}_{j} \leq \rho(t) \leq \hat{r}_{j}, \forall t \in[0, \sigma] \text { and } \rho(\sigma) \in\left\{\check{r}_{j}, \hat{r}_{j}\right\}
$$

We consider that case in which $\rho(\sigma)=\hat{r}_{j}$. The treatment of the other situation is completely symmetric (involving the consideration of $r_{-}$instead of $r_{+}$) and thus is omitted.

We want to prove that

$$
\theta(\sigma)-\theta(0)>2(j+1) \pi
$$

holds. Indeed, from (3.25) one can conclude easily by observing that $\theta(\tau)-\theta(\sigma)>-\pi$ (as a consequence of Lemma 3.1) and therefore $\theta(\tau)-\theta(0)>2 j \pi+\pi$, yielding (3.24).

If, by contradiction, we suppose that (3.25) is not true, from $\theta^{\prime}(t)>0$ for all $t \in[0, \sigma]$, we obtain

$$
0<\theta(t)-\theta(0)<2(j+1) \pi, \quad \forall t \in] 0, \sigma[.
$$

At this point we note that the first equation in (3.23) can be written as

$$
\rho^{\prime}=\rho S(t, \rho \cos \theta, \rho \sin \theta)
$$

where

$$
S(t, x, y):=(w(t, x)-x) \frac{y}{x^{2}+y^{2}},
$$

while the second equation in (3.23) is of the form

$$
\theta^{\prime}=U(t, \rho \cos \theta, \rho \sin \theta)
$$

with

$$
U(t, x, y):=\frac{y^{2}+w(t, x) x}{x^{2}+y^{2}} .
$$

Therefore, from the sign condition (3.11), we find that

$$
\frac{S(t, x, y)}{U(t, x, y)} \leq \mathscr{M}_{+}(x, y), \quad \forall t \in[0, \tau],|x| \leq \delta, y \in \mathbb{R}, x^{2}+y^{2}>0 .
$$

Let $\gamma: \mathbb{R} \rightarrow \mathbb{R}_{0}^{+}$be the solution of (3.18) with $r\left(\theta_{0}\right)=r_{j}^{*}$, that is $\gamma(\theta):=r_{+}\left(\theta ; \theta_{0}, r_{j}^{*}\right)$. From the definition of $M_{j}^{*}\left(\rho_{0}\right)$ and the choice of $\hat{r}_{j}$ in (3.19), we can find $\varepsilon>0$ such that

$$
\left.\gamma(\theta)<\hat{r}_{j}, \quad \forall \theta \in\right] \theta_{0}-\varepsilon, \theta_{0}+2(j+1) \pi+\varepsilon[.
$$


By the definition of $\gamma$ and (3.26) it follows that

$$
\left.R(t, \gamma(\theta), \theta) \leq \gamma^{\prime}(\theta) \Theta(t, \gamma(\theta), \theta), \quad \forall t \in[0, \tau], \theta \in\right] \theta_{0}-\varepsilon, \theta_{0}+2(j+1) \pi+\varepsilon[.
$$

Using Corollary 5.1 with the positions $\theta_{1}:=\theta_{0}-\varepsilon, \theta_{2}:=\theta_{0}+2(j+1) \pi+\varepsilon$ and $I:=[0, \sigma]$, we easily conclude that

$$
\rho(t) \leq \gamma(\theta(t)), \quad \forall t \in[0, \sigma]
$$

and hence

$$
\rho(t) \leq M_{j}^{*}<\hat{r}_{j} \quad \forall t \in[0, \sigma],
$$

thus contradicting the hypothesis that $\rho(\sigma)=\hat{r}_{j}$.

With a similar argument one can check that (3.25) holds if $\rho(\sigma)=\check{r}_{j}$.

Hence, in any case, (3.13) follows.

Remark 3.1 For the applications of our result it is crucial to observe that the constants $\tau_{j}^{*}$ and $r_{j}^{*}$ depend only on $j$, the comparison functions $g, h$ and the the size $|I|$ of the interval on which (3.11) is satisfied. Such constants do not depend on the particular function $w(t, x)$, in the sense that the same choice of $\tau_{j}^{*}$ and $r_{j}^{*}$ can be made for all the functions $w \in \mathcal{W}(g, h, I)$ and independently of the particular interval $I$.

For the next results we do not need to suppose that $w \in \mathcal{W}(g, h, I)$. However, we fix, from now on, a continuous function $w: \mathbb{R} \times \mathbb{R} \rightarrow \mathbb{R}$, with $w(t, 0) \equiv 0$, and assume the uniqueness and global continuability of the solutions of (3.12). Let also $J \subset \mathbb{R}$ be a fixed compact interval.

Before proceeding further, we make a short comment about the meaning of Lemma 3.1 and Lemma 3.2 with respect to the solutions of system (3.12). As observed along the proof of the above lemma, the result claimed in Lemma 3.1 automatically applies to (3.12) due to the particular form of the first equation in the system. It asserts that the angle associated to the solutions cannot turn back too much (the precise lower bound being given by $-1 / 2$ ). On the other hand, Lemma 3.2 asserts that, under (3.10) and (3.11) (conditions which "translate" $\left(f_{g, h}\right)$ to the setting of (3.12)), we can choose a circumference of initial points such that the corresponding solutions make a large number of turns if enough time is available. The next results show that, under conditions like $\left(f_{0}\right)$ (respectively, $\left.\left(f_{\infty}\right)\right)$, for any fixed time interval, we can find small solutions (respectively, large solutions) which cannot complete a turn. Similar results about rotation numbers can be already found in some previous papers (see $[5,7,13,43]$ ). We will present the details of the proofs for the reader's convenience.

Both Lemma 3.4 and Lemma 3.6 below (as well as their symmetric versions) rely on the following known estimate, the so called "elastic property" as it refers to the fact that solutions departing small (respectively, large) remain small (respectively, large) in a uniform manner. For a proof, see [7]

Lemma 3.3 In the above setting, there exist two continuous functions $\eta, v: \mathbb{R}_{0}^{+} \rightarrow \mathbb{R}_{0}^{+}$, with $\eta(s) \leq s \leq v(s)$, for all $s>0$ such that for every $\zeta(\cdot)$ solution of (3.12) we have:

$$
\min _{t \in J}|\zeta(t)| \leq \eta(r) \Longrightarrow \max _{t \in J}|\zeta(t)| \leq r
$$




$$
\max _{t \in J}|\zeta(t)| \geq v(R) \Longrightarrow \min _{t \in J}|\zeta(t)| \geq R .
$$

We consider at first the case of rotation numbers for small solutions.

Lemma 3.4 Suppose that

$$
\limsup _{x \rightarrow 0^{+}} \frac{w(t, x)}{x} \leq 0, \quad \text { uniformly in } t \in J .
$$

Then, there exists $r_{0}=r_{0}(w, J)>0$ such that

$$
\operatorname{Rot}(\zeta ; J)<1
$$

holds for every solution $\zeta(t)$ of (3.12) with $0<\min _{t \in J}|\zeta(t)| \leq r_{0}$. If, moreover, for some $t^{\prime} \in J$ it holds that $x\left(t^{\prime}\right) \geq 0$ and $y\left(t^{\prime}\right) \leq 0$, then

$$
\operatorname{Rot}\left(\zeta ; J^{\prime}\right)<1 / 4,
$$

for $J^{\prime}:=J \cap\left[t^{\prime},+\infty\right)$.

Proof. By (3.27) we have that for every $\varepsilon>0$ there exists $\delta_{\varepsilon}>0$ such that

$$
w(t, x) x \leq \varepsilon x^{2}, \quad \forall t \in J \text { and } 0 \leq x \leq \delta_{\varepsilon} .
$$

Let $\zeta(t)=(x(t), y(t))$ be a nontrivial solution of (3.12) satisfying

$$
|x(t)| \leq \delta:=\delta_{\varepsilon}, \quad \forall t \in J .
$$

Passing to the polar coordinates as in (3.22) we study the angular variation of the solution in order to show that the rotation number must be small if we take care of choosing $\varepsilon$ sufficiently small. To this aim, we prove the following claim which may have some independent interest (in particular, making more precise the argument used in [7, Lemma 3.2]).

Claim. Let us fix $\alpha \in] 0, \pi / 2\left[\right.$. Then there exists $\varepsilon^{*}=\varepsilon_{\alpha}^{*}>0$ such that, for each nontrivial solution satisfying (3.28) for $\varepsilon \leq \varepsilon^{*}$, the set $\theta(J)$ does not contain any interval of the form $\left[\theta_{1}, \theta_{2}\right]$ for some $\theta_{1}=\theta\left(t_{1}\right) \in[-\pi / 2+2 k \pi, 2 k \pi], \theta_{2}=\theta\left(t_{2}\right) \in[2 k \pi, \pi / 2+2 k \pi]$ (with $\left.k \in \mathbb{Z}\right)$, for $t_{1}<t_{2}$ and $\theta_{2}-\theta_{1}=\alpha$.

Suppose, by contradiction, that there exist $t^{\prime}, t^{\prime \prime} \in J\left(t^{\prime}<t^{\prime \prime}\right)$ and a pair $\theta_{1}, \theta_{2}$ as above such that

$$
\left.\theta\left(t^{\prime}\right)=\theta_{1}, \theta\left(t^{\prime \prime}\right)=\theta_{2} \quad \text { and } \theta(t) \in\right] \theta_{1}, \theta_{2}[, \forall t \in] t^{\prime}, t^{\prime \prime}[.
$$

By the choice of $\left[\theta_{1}, \theta_{2}\right]$, we have that $x(t)=\rho(t) \cos \theta(t) \in[0, \delta]$, for all $t \in\left[t^{\prime}, t^{\prime \prime}\right]$. Therefore, from (3.23), we have

$$
\theta^{\prime}(t) \leq \sin ^{2} \theta(t)+\varepsilon \cos ^{2} \theta(t), \quad \forall t \in\left[t^{\prime}, t^{\prime \prime}\right],
$$

that is

$$
\frac{\theta^{\prime}(t)}{\sin ^{2} \theta(t)+\varepsilon \cos ^{2} \theta(t)} \leq 1
$$


Let us introduce the auxiliary function

$$
\Psi(s):=\int_{0}^{s} \frac{d \xi}{\sin ^{2} \xi+\varepsilon \cos ^{2} \xi}, \quad \text { for } s \in \mathbb{R}
$$

so that (3.29) writes as

$$
\Psi^{\prime}(\theta(t)) \theta^{\prime}(t) \leq 1, \quad \forall t \in\left[t^{\prime}, t^{\prime \prime}\right] .
$$

An integration over $\left[t^{\prime}, t^{\prime \prime}\right]$ gives

$$
\Psi\left(\theta\left(t^{\prime \prime}\right)\right)-\Psi\left(\theta\left(t^{\prime}\right)\right) \leq \int_{t^{\prime}}^{t^{\prime \prime}} 1 d t \leq t^{\prime \prime}-t^{\prime} \leq|J|
$$

that is

$$
\int_{\theta_{1}=\theta\left(t^{\prime}\right)}^{\theta_{2}=\theta\left(t^{\prime \prime}\right)} \frac{d \theta}{\sin ^{2} \theta+\varepsilon \cos ^{2} \theta}=\Psi\left(\theta_{2}\right)-\Psi\left(\theta_{1}\right) \leq|J| .
$$

On the other hand,

$$
\int_{\theta_{1}}^{\theta_{2}} \frac{d \theta}{\sin ^{2} \theta+\varepsilon \cos ^{2} \theta}=\frac{1}{\sqrt{\varepsilon}}\left(\arctan \left(\varepsilon^{-1 / 2} \tan \theta_{2}\right)-\arctan \left(\varepsilon^{-1 / 2} \tan \theta_{1}\right)\right):=\phi_{\theta_{1}, \theta_{2}}(\varepsilon) .
$$

At this point, we recall that

$$
\tan \theta_{1} \leq 0 \leq \tan \theta_{2}, \quad \text { with } \theta_{2}-\theta_{1}=\alpha>0 .
$$

Then, an analysis for the search of the minimum of the function

$$
[-\alpha / 2, \alpha / 2] \mapsto \arctan \left(\varepsilon^{-1 / 2} \tan \left(s+\frac{\alpha}{2}\right)\right)-\arctan \left(\varepsilon^{-1 / 2} \tan \left(s-\frac{\alpha}{2}\right)\right)
$$

shows that

$$
\phi_{\theta_{1}, \theta_{2}}(\varepsilon) \geq \frac{1}{\sqrt{\varepsilon}} \min \left\{2 \arctan \left(\varepsilon^{-1 / 2} \tan \left(\frac{\alpha}{2}\right)\right), \arctan \left(\varepsilon^{-1 / 2} \tan (\alpha)\right)\right\} .
$$

The above minimum can be achieved by the first function or the second one, depending whether $0<\varepsilon<1$ or $\varepsilon>1$. In any case,

$$
\phi_{\theta_{1}, \theta_{2}}(\varepsilon) \rightarrow+\infty, \quad \text { for } \varepsilon \rightarrow 0^{+},
$$

uniformly on $\theta_{1} \leq 0$ and $\theta_{2} \geq 0$ with $\theta_{2}-\theta_{1}=\alpha$. In particular, if $0<\varepsilon<1$, we find

$$
\phi_{\theta_{1}, \theta_{2}}(\varepsilon) \geq \frac{\alpha}{\sqrt{\varepsilon}} .
$$

Thus a contradiction is achieved provided that $\varepsilon \in] 0,1\left[\right.$ is chosen so small that $\varepsilon<(\alpha /|J|)^{2}$. The Claim is proved. 
Let us fix now $\alpha=\pi / 4$ and take $0<\varepsilon \leq \varepsilon^{*}$. Suppose, by contradiction, that $\zeta(t)=$ $(x(t), y(t))$ is a nontrivial solution satisfying (3.28) and such that $\operatorname{Rot}(\zeta ; J) \geq 1$. Then, from the definition of rotation number (3.6), it follows that $\theta(\max J)-\theta(\min J) \geq 2 \pi$ and, consequently, the interval $[\theta(\min J), \theta(\max J)]($ contained in $\theta(J))$ must contain $(\bmod 2 \pi)$ at least one interval between $[-\pi / 4,0]$ and $[0, \pi / 4]$. This clearly contradicts the Claim (thanks to the choice of $\varepsilon$ ).

Finally, we invoke the elastic property and observe that the first part of Lemma 3.3 guarantees that the choice

$$
r_{0}:=\eta\left(\delta_{\varepsilon}\right)
$$

is adequate to conclude.

The proof of the second part of the lemma is omitted as it follows from the Claim using an analogous argument.

A symmetric version of our result reads as follows.

Lemma 3.5 Suppose that

$$
\limsup _{x \rightarrow 0^{-}} \frac{w(t, x)}{x} \leq 0, \quad \text { uniformly in } t \in J .
$$

Then, there exists $r_{0}=r_{0}(w, J)>0$ such that

$$
\operatorname{Rot}(\zeta ; J)<1
$$

holds for every solution $\zeta(t)$ of (3.12) with $0<\min _{t \in J}|\zeta(t)| \leq r_{0}$. If, moreover, for some $t^{\prime} \in J$ it holds that $x\left(t^{\prime}\right) \leq 0$ and $y\left(t^{\prime}\right) \geq 0$, then

$$
\operatorname{Rot}\left(\zeta ; J^{\prime}\right)<1 / 4,
$$

for $J^{\prime}:=J \cap\left[t^{\prime},+\infty\right)$.

We consider now the rotation numbers associated to large solutions.

Lemma 3.6 Suppose that

$$
\limsup _{x \rightarrow+\infty} \frac{w(t, x)}{x} \leq 0, \quad \text { uniformly in } t \in J .
$$

Then, there exists $R_{0}=R_{0}(w, J)>0$ such that

$$
\operatorname{Rot}(\zeta ; J)<1
$$

holds for every solution $\zeta(t)$ of (3.12) with $\max _{t \in J}|\zeta(t)| \geq R_{0}$. If, moreover, for some $t^{\prime} \in J$ it holds that $x\left(t^{\prime}\right) \geq 0$ and $y\left(t^{\prime}\right) \leq 0$, then

$$
\operatorname{Rot}\left(\zeta ; J^{\prime}\right)<1 / 4,
$$

for $J^{\prime}:=J \cap\left[t^{\prime},+\infty\right)$. 
Proof. By (3.31) we have that for every $\varepsilon>0$ there exists $M_{\varepsilon}>0$ such that

$$
w(t, x) x \leq \varepsilon x^{2}+M_{\varepsilon}, \quad \forall t \in J \text { and } x \geq 0 .
$$

Let $\zeta(t)=(x(t), y(t))$ be a nontrivial solution of (3.12) satisfying

$$
|\zeta(t)|^{2} \geq \frac{M_{\varepsilon}}{\varepsilon}, \quad \forall t \in J
$$

Passing again to the polar coordinates as in (3.22) we prove the following claim.

Claim. Let us fix $\alpha \in] 0, \pi / 2\left[\right.$. Then there exists $\varepsilon^{*}=\varepsilon_{\alpha}^{*}>0$ such that, for each solution satisfying (3.32) for $\varepsilon \leq \varepsilon^{*}$, the set $\theta(J)$ does not contain any interval of the form $\left[\theta_{1}, \theta_{2}\right]$ for some $\theta_{1}=\theta\left(t_{1}\right) \in[-\pi / 2+2 k \pi, 2 k \pi], \theta_{2}=\theta\left(t_{2}\right) \in[2 k \pi, \pi / 2+2 k \pi]$ (with $k \in \mathbb{Z}$ ), for $t_{1}<t_{2}$ and $\theta_{2}-\theta_{1}=\alpha$.

Suppose, by contradiction, that there exist $t^{\prime}, t^{\prime \prime} \in J\left(t^{\prime}<t^{\prime \prime}\right)$ and a pair $\theta_{1}, \theta_{2}$ as above such that

$$
\left.\theta\left(t^{\prime}\right)=\theta_{1}, \theta\left(t^{\prime \prime}\right)=\theta_{2} \quad \text { and } \theta(t) \in\right] \theta_{1}, \theta_{2}[, \forall t \in] t^{\prime}, t^{\prime \prime}[.
$$

By the choice of $\left[\theta_{1}, \theta_{2}\right]$, we have that $x(t)=\rho(t) \cos \theta(t) \geq 0$, for all $t \in\left[t^{\prime}, t^{\prime \prime}\right]$. Therefore, from (3.23), it holds that

$$
\theta^{\prime}(t) \leq \sin ^{2} \theta(t)+\varepsilon \cos ^{2} \theta(t)+\frac{M_{\varepsilon}}{\rho(t)^{2}} \quad t \in\left[t^{\prime}, t^{\prime \prime}\right]
$$

Using (3.32) for $0<\varepsilon<1$, so that $M_{\varepsilon} /|\zeta(t)|^{2} \leq \sin ^{2} \theta(t)+\varepsilon \cos ^{2} \theta(t)$, we obtain

$$
\theta^{\prime}(t) \leq 2\left(\sin ^{2} \theta(t)+\varepsilon \cos ^{2} \theta(t)\right) .
$$

From now on, the proof of the Claim proceeds like that of the analogous Claim in Lemma 3.4 and we skip the details. A contradiction is achieved provided that $\varepsilon \in] 0,1$ [ is chosen so small that $\varepsilon<(\alpha / 2|J|)^{2}$. The Claim is proved.

Let us fix now $\alpha=\pi / 4$ and take $0<\varepsilon \leq \varepsilon^{*}$. Suppose, by contradiction, that $\zeta(t)=$ $(x(t), y(t))$ is a nontrivial solution satisfying (3.32) and such that $\operatorname{Rot}(\zeta ; J) \geq 1$. Then, from the definition of rotation number (3.6), it follows that $\theta(\max J)-\theta(\min J) \geq 2 \pi$ and, consequently, the interval $[\theta(\min J), \theta(\max J)]($ contained in $\theta(J))$ must contain $(\bmod 2 \pi)$ at least one interval between $[-\pi / 4,0]$ and $[0, \pi / 4]$. This clearly contradicts the Claim (thanks to the choice of $\varepsilon$ ).

Finally, we invoke the elastic property and observe that the second part of Lemma 3.3 guarantees that the choice

$$
R_{0}:=v\left(\left(\frac{M_{\varepsilon}}{\varepsilon}\right)^{1 / 2}\right)
$$

is adequate to conclude.

The proof of the second part of the lemma is omitted as it follows from the Claim using an analogous argument.

Symmetrically, we also have 
Lemma 3.7 Suppose that

$$
\limsup _{x \rightarrow-\infty} \frac{w(t, x)}{x} \leq 0, \quad \text { uniformly in } t \in J .
$$

Then, there exists $R_{0}=R_{0}(w, J)>0$ such that

$$
\operatorname{Rot}(\zeta ; J)<1
$$

holds for every solution $\zeta(t)$ of (3.12) with $\max _{t \in J}|\zeta(t)| \geq R_{0}$. If, moreover, for some $t^{\prime} \in J$ it holds that $x\left(t^{\prime}\right) \leq 0$ and $y\left(t^{\prime}\right) \geq 0$, then

$$
\operatorname{Rot}\left(\zeta ; J^{\prime}\right)<1 / 4,
$$

for $J^{\prime}:=J \cap\left[t^{\prime},+\infty\right)$.

We end this section with some results which connect the rotation numbers associated to (3.12) with the nodal properties of the nontrivial solutions to the second order equation

$$
x^{\prime \prime}+w(t, x)=0, \quad t \in J:=\left[s_{0}, s_{1}\right]
$$

with $w(t, 0) \equiv 0$. For simplicity, we confine ourselves only to the cases of Dirichlet, Neumann and periodic boundary conditions. More general Sturm-Liouville type conditions can be considered as well, by suitably modifying the statements of the next lemma (see also $[9,38]$ for more details in this direction).

Lemma 3.8 Let $\zeta(t)=(x(t), y(t))$ be a (nontrivial) solution of (3.12) with

$$
\operatorname{Rot}(\zeta ; J)=\frac{k}{2}, \quad \text { for some positive integer } k .
$$

Then, the following conclusions hold:

- [Dirichlet BVP] If $x\left(s_{0}\right)=0$, then $x\left(s_{1}\right)=0$ and $x(\cdot)$ has exactly $k-1$ zeros in the interior of $J$.

- [Neumann BVP] If $x^{\prime}\left(s_{0}\right)=0$, then $x^{\prime}\left(s_{1}\right)=0$ and $x(\cdot)$ has exactly $k$ zeros in the interior of $J$.

- [Periodic BVP] If $x(\cdot)$ satisfies the periodic boundary conditions $x\left(s_{1}\right)-x\left(s_{0}\right)=$ $x^{\prime}\left(s_{1}\right)-x^{\prime}\left(s_{0}\right)=0$ and $k$ is even, then it has exactly $k$ zeros in $\left[s_{0}, s_{1}[\right.$.

Proof. From (3.12) we know that $x^{\prime}(t)=y(t) \neq 0$ for every $t$ such that $x(t)=0$. This immediately implies that all the zeros of $x(\cdot)$ are simple and thus in a finite number. Moreover, if $\left[t^{\prime}, t^{\prime \prime}\right] \subset\left[s_{0}, s_{1}\right]$ is any subinterval between two consecutive zeros, that is $x\left(t^{\prime}\right)=x\left(t^{\prime \prime}\right)=0$ and $x(t) \neq 0$ for all $t \in] t^{\prime}, t^{\prime \prime}\left[\right.$, then either $x^{\prime}\left(t^{\prime}\right)>0>x^{\prime}\left(t^{\prime \prime}\right)$ or $x^{\prime}\left(t^{\prime}\right)<0<x^{\prime}\left(t^{\prime \prime}\right)$. In both the cases, we have

$$
\begin{aligned}
2 \pi \operatorname{Rot}\left(\zeta ;\left[t^{\prime}, t^{\prime \prime}\right]\right) & =\int_{t^{\prime}}^{t^{\prime \prime}} \frac{d}{d t} \arctan \left(\frac{y(t)}{x(t)}\right) d t=-\int_{t^{\prime}}^{t^{\prime \prime}} \frac{d}{d t} \arctan \left(\frac{x^{\prime}(t)}{x(t)}\right) d t \\
& =-\left(\lim _{t \rightarrow\left(t^{\prime \prime}\right)^{-}} \arctan \left(x^{\prime}(t) / x(t)\right)-\lim _{t \rightarrow\left(t^{\prime}\right)^{+}} \arctan \left(x^{\prime}(t) / x(t)\right)\right)=\pi
\end{aligned}
$$


Therefore, the rotation counts as $1 / 2$ on each subinterval of two consecutive zeros. With a similar computation we can check that if if $\left[t^{\prime}, s^{\prime}\right] \subset\left[s_{0}, s_{1}\right]$ is any subinterval such that $x\left(t^{\prime}\right)=0$ and $x(t) \neq 0$ for all $\left.\left.t \in\right] t^{\prime}, s^{\prime}\right]$, then

$$
0<2 \pi \operatorname{Rot}\left(\zeta ;\left[t^{\prime}, s^{\prime}\right]\right)<\pi .
$$

Suppose that $x\left(s_{0}\right)=0$. Since $2 \pi \operatorname{Rot}(\zeta ; J)=k \pi$, we know that $x(t)$ vanishes at least once on $\left.] s_{0}, s_{1}\right]$. Assume that $x(\cdot)$ has, besides $s_{0}$ precisely other $j$ (simple) zeros $\tau_{1} \ldots, \tau_{j}$ that we ca order as follows $s_{0}:=\tau_{0}<\tau_{1}<\cdots<\tau_{j} \leq s_{1}$. In this case, we conclude that

$$
\operatorname{Rot}(\zeta ; J)=\sum_{i=1}^{j} \operatorname{Rot}\left(\zeta ;\left[\tau_{i-1}, \tau_{i}\right]\right)+\operatorname{Rot}\left(\zeta ;\left[\tau_{j}, s_{1}\right]\right)=\frac{j}{2}+\operatorname{Rot}\left(\zeta ;\left[\tau_{j}, s_{1}\right]\right) .
$$

If $\tau_{j}<s_{1}$, we conclude that $j / 2<\operatorname{Rot}(\zeta ; J)<(j+1) / 2$, which is in contrast with the assumption that $2 \operatorname{Rot}(\zeta ; J)$ is an integer. Therefore, $\tau_{j}=s_{1}$ and $j=k$. Hence $x(t)$ satisfies the Dirichlet boundary condition $x\left(s_{1}\right)=x\left(s_{0}\right)=0$, it has exactly $k+1$ zeros in $J$ and so $k-1$ simple zeros in the interior of $J$. The first assertion is proved.

Suppose now $x^{\prime}\left(s_{0}\right)=0$. If, by contradiction, $x(t)$ never vanishes, then we have

$$
\left.2 \pi \operatorname{Rot}(\zeta ; J)=\arctan \left(x^{\prime}\left(s_{0}\right) / x\left(s_{0}\right)\right)-\arctan \left(x^{\prime}\left(s_{1}\right) / x\left(s_{1}\right)\right) \in\right]-\pi / 2, \pi / 2[
$$

which is in contrast with the fact that $k$ is a positive integer. Hence, we can assume that $x(\cdot)$ has $j$ (simple) zeros $\tau_{1} \ldots, \tau_{j}$ that we can order as follows $s_{0}<\tau_{1}<\cdots<\tau_{j} \leq s_{1}$. Repeating again the same computation as above and using the fact that

$$
\operatorname{Rot}\left(\zeta ;\left[\tau_{1}, \tau_{j}\right]\right)=\sum_{i=1}^{j-1} \operatorname{Rot}\left(\zeta ;\left[\tau_{i}, \tau_{i+1}\right]\right)=\frac{j-1}{2},
$$

we find

$$
\begin{aligned}
2 \pi \operatorname{Rot}(\zeta ; J) & =\int_{s_{0}}^{\tau_{1}} \frac{d}{d t} \arctan \left(\frac{-x^{\prime}(t)}{x(t)}\right) d t+\pi(j-1)+\int_{\tau_{j}}^{s_{1}} \frac{d}{d t} \arctan \left(\frac{-x^{\prime}(t)}{x(t)}\right) d t \\
& =\frac{\pi}{2}+\pi(j-1)+\chi,
\end{aligned}
$$

where $\chi:=\int_{\tau_{j}}^{s_{1}} \frac{d}{d t} \arctan \left(\frac{-x^{\prime}(t)}{x(t)}\right) d t \in[0, \pi[$, by (3.35). Note that $\chi=0$ if and only if $\tau_{j}=s_{1}$. On the other hand, from $\operatorname{Rot}(\zeta ; J)=\frac{k}{2}$ we find that

$$
\frac{1}{4}+\frac{j-1}{2}+\frac{\chi}{2 \pi}=\frac{k}{2}
$$

and hence $\chi>0$. Then we have $\tau_{j}<s_{1}$ and therefore $\chi=\frac{\pi}{2}-\arctan \left(x^{\prime}\left(s_{1}\right) / x\left(s_{1}\right)\right)$. A comparison with the above relation yields

$$
\frac{j}{2}=\frac{k}{2}+\frac{\arctan \left(x^{\prime}\left(s_{1}\right) / x\left(s_{1}\right)\right)}{2 \pi} .
$$


As a consequence, $x^{\prime}\left(s_{1}\right)=0$ and $k=j$. We have thus found that the solution satisfies the Neumann boundary condition $x^{\prime}\left(s_{0}\right)=x^{\prime}\left(s_{1}\right)=0$ with precisely $k$ zeros in the interior of the interval.

The proof for the periodic case follows a completely similar argument and thus is omitted (see [37] for more details in the periodic setting).

\subsection{Proofs}

In this section we apply the previous lemmas to a system of the form (3.12) which comes from (3.1) after a change in the independent variable. More precisely, for any $\lambda>0$, we set

$$
w(t, x, \lambda):=f\left(\frac{t}{\sqrt{\lambda}}, x\right), \quad t \in[0, \sqrt{\lambda} T], \quad x \in \mathbb{R} .
$$

In order to enter in the setting of the preceding subsection we also extend (in the obvious way) $w(\cdot, x)$ to $\mathbb{R}$. Observe that if $(x(t), y(t))$ is a solution of

$$
\left\{\begin{array}{l}
x^{\prime}=-y \\
y^{\prime}=w(t, x, \lambda)
\end{array}\right.
$$

with $\left(x\left(t_{0}\right), y\left(t_{0}\right)\right)=\left(x_{0}, y_{0}\right)$ for some $t_{0} \in[0, \sqrt{\lambda} T]$, then $u(t):=x(\sqrt{\lambda} t)$, with $t \in[0, T]$, is a solution of (3.1) with $u\left(t_{0} / \sqrt{\lambda}\right)=x_{0}$ and $u^{\prime}\left(t_{0} / \sqrt{\lambda}\right)=-\sqrt{\lambda} y_{0}$. All the hypotheses on $f(t, x)$ now are transferred to the function $w(t, x, \lambda)$. In particular, $\left(f_{0}\right)$ and $\left(f_{\infty}\right)$ read exactly the same (with $f$ replaced by $w$ ), uniformly for $t \in[0, \sqrt{\lambda} T]$, while $\left(f_{g, h}\right)$ now rewrites with (3.2) replaced by

$$
g(x) x \leq w(t, x, \lambda) \leq h(x) x, \quad \text { for every } t \in \sqrt{\lambda} I_{0}=[\sqrt{\lambda} a, \sqrt{\lambda} b],|x| \leq \delta,
$$

for the same functions $g, h$ as in $\left(f_{g, h}\right)$.

Now we are in position to start with the proofs. In order to simplify the notation, in what follows, all the rotation numbers refer to the solutions of (3.36). We also denote by $\zeta(\cdot ; s, z)$ the solution $(x(t), y(t))$ of system (3.36) with $(x(s), y(s))=z$.

Proof. [Proof of Theorem 3.1]. First of all, given any positive integer $m$, we define

$$
\lambda_{m}^{*}:=\left(\frac{\tau_{\ell(m)}^{*}}{\left|I_{0}\right|}\right)^{2}
$$

where

$$
\ell(m):=1+\left\lceil\frac{m+1}{2}\right\rceil
$$

and $\tau_{\ell(m)}^{*}$ is the constant given by Lemma 3.2.

Now we fix $\lambda>\lambda_{m}^{*}$ and verify the statement of the theorem, by applying Lemma 3.2 with $I:=\sqrt{\lambda} I_{0}$ and the subsequent lemmas with $J:=[0, \sqrt{\lambda} T]$.

By the choice of $\lambda_{m}^{*}$ we have that

$$
\operatorname{Rot}(\zeta ;[\sqrt{\lambda} a, \sqrt{\lambda} b])=\operatorname{Rot}(\zeta ; I)>\ell(m) \geq \frac{m+1}{2}+1, \quad \forall z_{0} \text { with }\left|z_{0}\right|=r_{\ell(m)}^{*},
$$


where $\zeta(t):=\zeta\left(t ; \sqrt{\lambda} a, z_{0}\right)$.

Let $\left(0, y^{*}\right)$; with $y^{*}<0$ be a point on the negative $y$-axis such that

$$
\left|\zeta\left(\sqrt{\lambda} a ; 0,\left(0, y^{*}\right)\right)\right|=r_{\ell(m)}^{*} .
$$

Note that such a point always exists by an elementary continuity argument. Using Lemma 3.1 we find

$$
\begin{aligned}
\operatorname{Rot}\left(\left(0, y^{*}\right) ; J\right) & =\operatorname{Rot}\left(\left(0, y^{*}\right) ;[0, \sqrt{\lambda} T]\right) \\
& =\operatorname{Rot}\left(\zeta^{*} ;[0, \sqrt{\lambda} a]\right)+\operatorname{Rot}\left(\zeta^{*} ;[\sqrt{\lambda} a, \sqrt{\lambda} b]\right)+\operatorname{Rot}\left(\zeta^{*} ;[\sqrt{\lambda} b, \sqrt{\lambda} T]\right) \\
& >-\frac{1}{2}+\frac{m+1}{2}+1-\frac{1}{2}=\frac{m+1}{2} \geq 1,
\end{aligned}
$$

where we have set

$$
\zeta^{*}(t):=\zeta\left(t ; 0,\left(0, y^{*}\right)\right)
$$

Assumption $\left(f_{0}\right)$ permits to apply Lemma 3.4 or Lemma 3.5. In any case, there exists $r_{0}>0$ such that for every $(0, y)$ with $0<|y| \leq r_{0}$ it holds:

$$
\operatorname{Rot}((0, y) ; J)<1 \text {. }
$$

Note that $r_{0}$ depends on $\lambda$ and, moreover, $r_{0}<\left|y^{*}\right|$.

The continuity of the rotation number ensures the existence of at least $m$ points

$$
y^{*}<y_{m}<y_{m-1}<\cdots<y_{\ell}<y_{\ell-1}<\ldots y_{1}<0,
$$

such that, for every $j=1, \ldots, m$, we have

$$
\operatorname{Rot}\left(\left(0, y_{j}\right) ; J\right)=\frac{j+1}{2}
$$

Hence, Lemma 3.8 applied to the solution $\zeta_{j}(t)=\left(x_{j}(t), y_{j}(t)\right):=\zeta\left(t ; 0,\left(0, y_{j}\right)\right)$, implies that $x_{j}(\sqrt{\lambda} T)=0$ and the existence of precisely $j$ simple zeros for $x_{j}(t)$ in $] 0, \sqrt{\lambda} T[$.

In this manner, for every $j=1, \ldots, m$ we have found at least one small solution $u_{j}(t)$ of the Dirichlet problem (3.4) with positive slope at $t=0$ and having precisely $j$ zeros in ] $0, T[$.

The $m$ small solutions with negative slope at $t=0$ and having, respectively, $1,2, \ldots, m$ simple zeros in $] 0, T$ [ can be found using a symmetric argument, that is, by shooting from the positive $y$-axis.

On the other hand, assumption $\left(f_{\infty}\right)$ permits to apply Lemma 3.6 or Lemma 3.7. In any case, there exists $R_{0}>0$ such that for every $(0, y)$ with $|y| \geq R_{0}$ it holds:

$$
\operatorname{Rot}((0, y) ; J)<1 \text {. }
$$

Also the constant $R_{0}$ depends on $\lambda$. Moreover, we have $R_{0}>\left|y^{*}\right|$.

The continuity of the rotation number ensures the existence of at least $m$ points

$$
\check{y}_{1}<\cdots<\check{y}_{\ell-1}<\check{y}_{\ll}<\cdots<\check{y}_{m}<y^{*}<0
$$


such that, for every $j=1, \ldots, m$, we have

$$
\operatorname{Rot}\left(\left(0, \check{y}_{j}\right) ; J\right)=\frac{j+1}{2} .
$$

Hence, Lemma 3.8 applied to the solution $\check{\zeta}_{j}(t)=\left(\check{x}_{j}(t), \check{y}_{j}(t)\right):=\zeta\left(t ; 0,\left(0, \check{y}_{j}\right)\right)$, implies that $\check{x}_{j}(\sqrt{\lambda} T)=0$ and the existence of precisely $j$ simple zeros for $\check{x}_{j}(t)$ in $] 0, \sqrt{\lambda} T[$.

Therefore, for every $j=1, \ldots, m$ we have found at least one large solution $\check{u}_{j}(t)$ of the Dirichlet problem (3.4) with positive slope at $t=0$ and having precisely $j$ zeros in $] 0, T[$.

The $m$ large solutions with negative slope at $t=0$ and having, respectively, $1,2, \ldots, m$ simple zeros in ]0, $T$ [ can be found using a symmetric argument, that is, by shooting from the positive $y$-axis.

Summarizing, we have found small solutions and large solutions of (3.7) with positive and, respectively, negative slope at $t=0$, satisfying the Dirichlet boundary condition $u(0)=0=u(T)$. They are at least $4 m$ and, for each $j=1, \ldots, m$ there are at least four with precisely $j$ zeros in ]0, $T$ [, namely, a small solution and a large solution with positive initial slope as well as a a small solution and a large solution with negative initial slope.

Proof. [Proof of Theorem 3.4]. We define

$$
\bar{\lambda}_{0}:=\lambda_{1}^{*}
$$

with $\lambda_{1}^{*}$ defined as in the proof of Theorem 3.1. Actually, a sharper constant could be found. However, we prefer such a choice since, in this manner, we can take advantage of various steps already developed in the preceding proof.

Just to fix the ideas, let us assume $\left(f_{0}^{+}\right)$. Keeping all the notation previously introduced, we have that

$$
\operatorname{Rot}\left(\left(0, y^{*}\right) ; J\right)>1
$$

for a suitable $y^{*}<0$. On the other hand, from the second part of Lemma 3.4 there exists $r_{0}>0$ such that for every $(0, y)$ with $-r_{0} \leq y<0$, it holds that

$$
\operatorname{Rot}((0, y) ; J)<1 / 4 \text {. }
$$

By continuity, we find at least one point $\left.y_{0} \in\right] y^{*}, 0[$ such that

$$
\operatorname{Rot}\left(\left(0, y_{0}\right) ; J\right)=1 / 2 \text {. }
$$

Hence, Lemma 3.8 applied to the solution $\zeta_{0}(t)=\left(x_{0}(t), y_{0}(t)\right):=\zeta\left(t ; 0,\left(0, y_{0}\right)\right)$, implies that $x_{0}(t)$ satisfies the Dirichlet boundary conditions on $[0, \sqrt{\lambda} T]$ and never vanishes in the interior of the interval. The corresponding solution $u_{0}(t)=x_{0}(t \sqrt{\lambda})$, for $t \in[0, T]$, is a positive solution of problem (3.4) (indeed, it starts with positive slope and is without internal zeros).

With a symmetric argument, we can see that when $\left(f_{0}^{-}\right)$, holds, we can start from the positive $y$-axis (this means shooting with negative initial slope) in order to find a negative solution of (3.4).

Finally, if $\left(f_{\infty}^{+}\right)$(respectively, $\left.\left(f_{\infty}^{-}\right)\right)$holds, we can obtain a large positive solution (a large negative solution, respectively), using the same technique. 
Proof. [Proof of Theorem 3.3]. The existence and multiplicity of periodic solutions will be provided by an application of the Poincaré-Birkhoff fixed point theorem to the Poincaré operator

$$
\Phi: z \mapsto \Phi(z):=\zeta\left(t_{0}+\sqrt{\lambda} T ; t_{0}, z\right) .
$$

With this respect it will be convenient to extend $w(\cdot, x, \lambda)$ to the whole real line by periodicity (more precisely, by $\sqrt{\lambda} T$-periodicity). Such an extension is harmless since in the worst case it will produce a discontinuity only at the points $(t, x, \lambda)$ with $t=k \sqrt{\lambda} T$ for $k \in \mathbb{Z}$. Such possible discontinuities are easily handled if we consider the solutions in the Carathéodory sense [20]. The situation is even better if $f(0, x)=f(T, x)$. In this case, the above extension is always continuous.

For a positive integer $m$, let us define

$$
\Lambda_{m}^{*}:=\left(\frac{\tau_{m+1}^{*}}{\left|I_{0}\right|}\right)^{2},
$$

where $\tau_{m+1}^{*}$ is the constant given by Lemma 3.2.

Now we fix $\lambda>\Lambda_{m}^{*}$ and consider the operator $\Phi$ for $t_{0}=\sqrt{\lambda} a$. We shall apply Lemma 3.2 with $I:=\sqrt{\lambda} I_{0}$ and the subsequent lemmas with $J:=[\sqrt{\lambda} a, \sqrt{\lambda} a+\sqrt{\lambda} T]$.

By the choice of $\Lambda_{m}^{*}$ we have that

$$
\operatorname{Rot}(\zeta ;[\sqrt{\lambda} a, \sqrt{\lambda} b])=\operatorname{Rot}(\zeta ; I)>m+1, \quad \forall z_{0} \text { with }\left|z_{0}\right|=r_{m+1}^{*},
$$

where $\zeta(t):=\zeta\left(t ; \sqrt{\lambda} a, z_{0}\right)$.

Using Lemma 3.1, for every $\left|z_{0}\right|=r_{m+1}^{*}$, we find

$$
\begin{aligned}
\operatorname{Rot}\left(z_{0} ; J\right) & =\operatorname{Rot}\left(z_{0} ;[\sqrt{\lambda} a, \sqrt{\lambda} a+\sqrt{\lambda} T]\right) \\
& =\operatorname{Rot}\left(z_{0} ;[\sqrt{\lambda} a, \sqrt{\lambda} b]\right)+\operatorname{Rot}(\zeta ;[\sqrt{\lambda} b, \sqrt{\lambda} a+\sqrt{\lambda} T])>(m+1)-\frac{1}{2}>m
\end{aligned}
$$

Assumption $\left(f_{0}\right)$ permits to apply Lemma 3.4 or Lemma 3.5. In any case, there exists $r_{0}>0$ such that for every $z_{0}$ with $0<\left|z_{0}\right| \leq r_{0}$ it holds:

$$
\operatorname{Rot}\left(z_{0} ; J\right)<1 .
$$

Note that $r_{0}$ depends on $\lambda$ and, moreover, $r_{0}<r_{m+1}^{*}$.

The Poincaré-Birkhoff fixed point theorem (cf. Theorem 5.1) ensures, for every $j=$ $1, \ldots, m$, the existence of at least two fixed points $z_{1}^{j}$ and $z_{2}^{j}$ for $\Phi$ in the open annular region $r_{0}<|z|<r_{m+1}^{*}$, such that

$$
\operatorname{Rot}\left(z_{1}^{j} ; J\right)=\operatorname{Rot}\left(z_{2}^{j} ; J\right)=j .
$$

Hence, Lemma 3.8 applied to the corresponding solutions $\zeta_{i, j}(t)=\left(x_{i, j}(t), y_{i, j}(t)\right):=\zeta\left(t ; t_{0}, z_{i}^{j}\right)$ (with $i=1,2$ ), implies the existence of precisely $2 j$ simple zeros for $x_{i, j}(t)$ in $\left[t_{0}, t_{0}+\sqrt{\lambda} T[\right.$.

In this manner, for every $j=1, \ldots, m$ we have found at least two small $T$-periodic solution $u_{1, j}(t)$ and $u_{2, j}(t)$ of (3.1) having precisely $2 j$ zeros in $\left[t_{0}, t_{0}+T[\right.$ as well as in $[0, T[$. 
On the other hand, assumption $\left(f_{\infty}\right)$ permits to apply Lemma 3.6 or Lemma 3.7. In any case, there exists $R_{0}>0$ such that for every $z_{0}$ with $\left|z_{0}\right| \geq R_{0}$ it holds:

$$
\operatorname{Rot}\left(z_{0} ; J\right)<1 .
$$

Also the constant $R_{0}$ depends on $\lambda$. Moreover, we have $R_{0}>r_{m+1}^{*}$.

The Poincaré-Birkhoff fixed point theorem ensures, for every $j=1, \ldots, m$, the existence of at least two fixed points $\breve{z}_{1}^{j}$ and $\breve{z}_{2}^{j}$ for $\Phi$ in the open annular region $r_{m+1}^{*}<|z|<R_{0}$, such that

$$
\operatorname{Rot}\left(\check{z}_{1}^{j} ; J\right)=\operatorname{Rot}\left(\check{z}_{2}^{j} ; J\right)=j .
$$

Hence, Lemma 3.8 applied to the corresponding solutions $\check{\zeta}_{i, j}(t)=\left(\check{x}_{i, j}(t), \check{y}_{i, j}(t)\right):=\zeta\left(t ; t_{0}, \breve{z}_{i}^{j}\right)$ (with $i=1,2$ ), implies the existence of precisely $2 j$ simple zeros for $\check{x}_{i, j}(t)$ in $\left[t_{0}, t_{0}+\sqrt{\lambda} T[\right.$.

In this manner, for every $j=1, \ldots, m$ we have found at least two large $T$-periodic solution $\check{u}_{1, j}(t)$ and $\breve{u}_{2, j}(t)$ of (3.1) having precisely $2 j$ zeros in $\left[t_{0}, t_{0}+T[\right.$ as well as in $[0, T[$.

Summarizing, we have found small solutions and large solutions of (3.7). They are at least $4 m$ and, for each $j=1, \ldots, m$ there are at least four with precisely $2 j$ zeros in $[0, T[$, namely, two small solution and two large solution.

\section{Variants of the main results, remarks and applications}

In this section we present some possible applications of the main results. We also propose certain variants of our theorems which can be obtained via minor modifications of the arguments.

The proofs of the theorems in Section 3 depend on the uniqueness and global continuability of the solutions of the Cauchy problems associated to (3.1). First of all we make some brief comments which will allow us to present a few results where these assumptions will be no more explicitly required.

The assumption about the uniqueness of the solutions is crucial for the proof of Theorem 3.3 as it guarantees the correct definition of the Poincaré map $\Phi$ as a global homeomorphism of the plane. On the other hand, for the Sturm-Liouville problems a "shooting without uniqueness" technique can be suitably implemented (see [9] and [10] for two alternative approaches in this direction). In any case, however, one has to suppose that $f(t, 0) \equiv 0$ and that a nontrivial solution of (3.36) never reaches the origin. For simplicity in all our applications we shall assume a local Lipschitz continuity for $f(t, x)$ in the $x$-variable. We notice, however, that such an hypothesis could be largely relaxed.

Concerning the global continuability of the solutions, we observe that it will be automatically satisfied (either directly, or after performing a truncation procedure) whenever we take some sign or growth assumptions which are pretty natural in the context of the problems with sublinear growth at infinity. In particular, we can put some conditions on $f(t, x)$ which guarantee, at the same time, both the global continuability and $\left(f_{\infty}\right)$. Just to start with an example in this direction, consider the following Landesman-Lazer type condition: 
$(L L) \quad$ Let $d>0$ and let $\gamma_{1}, \gamma_{2}:[0, T] \rightarrow \mathbb{R}$ be continuous functions with

$$
\int_{0}^{T} \gamma_{1}(t) d t \leq 0 \leq \int_{0}^{T} \gamma_{2}(t) d t
$$

such that, for every $t \in[0, T]$, it holds that

$$
f(t, x) \leq \gamma_{1}(t) \text { for all } x \leq-d \text { and } f(t, x) \geq \gamma_{2}(t) \text { for all } x \geq d .
$$

As proved in $[13,12]$, condition $(L L)$, when paired with a one-sided sublinear growth at infinity (in particular, $\left(f_{\infty}\right)$ is enough), guarantees the global continuability of the solutions.

In the special case when

$$
f(t, x)=q(t) f(x),
$$

with $f: \mathbb{R} \rightarrow \mathbb{R}$ locally Lipschitz and $q:[0, T] \rightarrow \mathbb{R}$ continuous, the following consequences of our main results can be produced for the equation

$$
u^{\prime \prime}+\lambda q(t) \mathrm{f}(u)=0 .
$$

Corollary 4.1 Suppose that

$$
\begin{gathered}
\mathrm{f}(x) x>0, \quad \forall x \neq 0, \\
\lim _{x \rightarrow 0^{+}} \frac{\mathrm{f}(x)}{x}=0 \quad \text { or } \quad \lim _{x \rightarrow 0^{-}} \frac{f(x)}{x}=0
\end{gathered}
$$

and

$$
\lim _{x \rightarrow+\infty} \frac{f(x)}{x}=0 \quad \text { or } \lim _{x \rightarrow-\infty} \frac{f(x)}{x}=0 .
$$

If, moreover, $q(t) \geq 0$ for all $t \in[0, T]$, with $q \not \equiv 0$, then the same conclusions of Theorems 3.1, 3.2 and 3.3 hold for equation (4.2).

Proof. By the assumptions, it is obvious that $\left(f_{0}\right)$ and $\left(f_{\infty}\right)$ hold for $f(t, x)$ as in (4.1). Notice that the global continuability of the solutions is satisfied because $f(t, x)$ satisfies a $(L L)$-type condition with $\gamma_{1}=\gamma_{2} \equiv 0$. The sign condition $\left(f_{g . h}\right)$ is fulfilled as explained at the beginning of Section 3.

The assumption on $\mathrm{f}(x) / x$ at $\pm \infty$ could be replaced by

$$
\lim _{x \rightarrow+\infty} \frac{\mathrm{F}(x)}{x^{2}}=0 \quad \text { or } \lim _{x \rightarrow-\infty} \frac{\mathrm{F}(x)}{x^{2}}=0,
$$

for $F(x):=\int_{0}^{x} f(\xi) d \xi$ (in this case, instead of using Lemma 3.6 and Lemma 3.7, the proof that large solutions have small rotation numbers follows from a result in [13]).

Corollary 4.1 extends to (4.1) the results obtained in Section 2 for the autonomous equation $u^{\prime \prime}+\lambda f(u)=0$.

Examples of equations to which Corollary 4.1 applies are given, for instance, by

$$
u^{\prime \prime}+\lambda q(t)|u|^{p}(\exp u-1)=0,
$$

for $p>1$ and with $q(t) \geq 0$ and not identically zero.

Our next result is of non-uniform nature, in the sense that we consider only a local sign condition on $f$ and we also allow $q(t)$ to change its sign. 
Corollary 4.2 Suppose that

$$
\exists \delta>0: \quad x \mathrm{f}(x)>0, \quad \forall x: 0<|x| \leq \delta
$$

and

$$
\lim _{x \rightarrow 0^{+}} \frac{f(x)}{x}=0 \quad \text { or } \lim _{x \rightarrow 0^{-}} \frac{f(x)}{x}=0 .
$$

Let also exist $x_{*}<-\delta$ and $x^{*}>\delta$ such that

$$
\mathrm{f}\left(x_{*}\right)=\mathrm{f}\left(x^{*}\right)=0 .
$$

Then, if $\max _{t \in[0, T]} q(t)>0$, the same conclusions of Theorems 3.1, 3.2 and 3.3 for equation (4.2) and all the corresponding solutions take value in the open interval $] x_{*}, x^{*}[$.

Proof. First of all, we define $\tilde{\mathrm{f}}(s):=\mathrm{f}\left(\min \left\{x^{*}, \max \left\{x_{*}, s\right\}\right\}\right)$, so that $\tilde{\mathrm{f}} \equiv \mathrm{f}$ on $\left[x_{*}, x^{*}\right]$ and $\tilde{\mathrm{f}}$ vanishes on $\mathbb{R} \backslash\left[x_{*}, x^{*}\right]$. We look for the solutions of the truncated equation

$$
u^{\prime \prime}+\lambda \tilde{f}(t, u)=0,
$$

for $\tilde{f}(t, x):=q(t) \tilde{f}(x)$. The uniqueness and global continuability of the solutions for (4.3) are obviously guaranteed. Since $\tilde{\mathrm{f}}(x)=\mathrm{f}(x)$ for $|x| \leq \delta$ and $\max _{t \in[0, T]} q(t)>0$, we have $\left(f_{g, h}\right)$ and $\left(f_{0}\right)$ satisfied. The assumption at infinity trivially holds as $\tilde{f}(t, x)=0$ for $|x|$ large. Then Theorems 3.1, 3.2 and 3.3 apply to (4.3). An elementary direct argument ensures that the solutions we find take values in $] x_{*}, x^{*}[$ and hence they are solutions of equation (4.2) as well.

Examples of equations to which Corollary 4.2 applies are given, for instance, by

$$
u^{\prime \prime}+\lambda q(t)|u|^{p} \sin u=0,
$$

for $p>1$ and with $\max _{[0, t]} q(t)>0$. The solutions we find are all with range in $]-\pi, \pi[$.

Corollary 4.2 is a simplified version of a more general result that could be obtained for equation (3.1). Indeed, if for an arbitrary function $f(t, x)$ (not necessarily of the form (4.1)) we have that there exist

$$
x_{*}<0<x^{*}
$$

such that

$$
f\left(t, x_{*}\right) \geq 0 \geq f\left(t, x^{*}\right), \quad \forall t \in[0, T],
$$

then we can repeat a similar truncation as above and obtain that both the global continuability and $\left(f_{\infty}\right)$ are satisfied for the corresponding truncated equation. Moreover, the solutions with the properties claimed in Theorems 3.1, 3.2 and 3.3 will have range in the open interval $] x_{*}, x^{*}[$ and thus will solve the original equation, too. Such an observation applies in particular to condition (1.7) in Rabinowitz theorem [34, Theorem 3] where we have $f(t, x) / x<0$ for all $t \in[0, T]$ and every $|x|$ large.

The constant functions $\alpha(t) \equiv x_{*}$ and $\beta(t) \equiv x^{*}$ are, respectively, a negative lower solution and a positive upper solution for the Dirichlet, Neumann and periodic boundary value problems associated with (3.1). Similar results can be obtained for non-constant 
lower and upper solutions. See also [8] for recent results where similar considerations have been developed in connection with the study of subharmonic solutions for some second order ODEs. In this more general setting, we can apply our theorems of Section 3 to equations like

$$
u^{\prime \prime}+\lambda\left(q(t)|u|^{p-1} u-\rho(t)|u|^{\gamma-1} u\right)=0
$$

when $1<p<\gamma, \max _{[0, T]} q(t)>0$, and $\min _{[0, T]} \rho(t)>0$.

\section{Appendix}

\subsection{A version of the Poincaré-Birkhoff fixed point theorem}

We consider a planar Hamiltonian system

$$
x^{\prime}=-\frac{\partial}{\partial y} \mathcal{H}(t, x, y), \quad y^{\prime}=\frac{\partial}{\partial x} \mathcal{H}(t, x, y)
$$

and suppose that the vector field $\vec{F}(t, z):=\left(-\frac{\partial}{\partial y} \mathcal{H}(t, x, y), \frac{\partial}{\partial x} \mathcal{H}(t, x, y)\right)$, for $z=(x, y)$, is continuous and $T$-periodic in the $t$-variable. We also assume the uniqueness and the global continuability for the solutions of the initial value problems. Let us fix $t_{0} \in[0, T[$. For any initial point $z \in \mathbb{R}^{2}$ we denote bt $\zeta\left(\cdot ; t_{0}, z\right)=\left(\zeta_{1}\left(\cdot ; t_{0}, z\right), \zeta_{2}\left(\cdot ; t_{0}, z\right)\right)$ the solution of $(5.1)$ with $\zeta\left(t_{0} ; t_{0}, z\right)=z$. If

$$
\vec{F}(t, 0) \equiv 0,
$$

we have that $\zeta\left(t ; t_{0}, z\right) \neq 0$ for every $t$, provided that $z \neq 0$. Hence we can pass to polar coordinates and define the rotation number associated to the solution $\zeta(t)=\zeta\left(t ; t_{0}, z\right)$ on a time interval $\left[t_{0}, t_{0}+\tau\right]$, as

$$
\operatorname{Rot}_{(5.1)}\left(z ;\left[t_{0}, t_{0}+\tau\right]\right):=\frac{1}{2 \pi} \int_{0}^{\tau} \frac{\frac{\partial}{\partial y} \mathcal{H}(t, \zeta(t)) \zeta_{2}(t)+\frac{\partial}{\partial x} \mathcal{H}(t, \zeta(t)) \zeta_{1}(t)}{\zeta_{1}(t)^{2}+\zeta_{2}(t)^{2}} d t
$$

Using the fact that (as a consequence of Liouville's theorem), for every $\tau>0$, the mapping $\Phi^{\tau}: z \mapsto \zeta\left(t_{0}+\tau ; t_{0}, z\right)$ is an area preserving homeomorphism of the plane onto itself, satisfying

$$
\Phi^{\tau}(0)=0,
$$

we can apply a consequence of the Poincaré-Birkhoff fixed point theorem which reads as follows (compare also with [26]).

Theorem 5.1 Assume (5.2) and let $k \geq 1$ be a fixed integer. Suppose that there are $0<$ $r_{0}<R_{0}$ and a positive integer $j$ such that

$$
\begin{aligned}
& \operatorname{Rot}_{(5.1)}\left(z ;\left[t_{0}, t_{0}+k T\right]\right)<j, \forall|z|=r_{0} \text { and } \operatorname{Rot}_{(5.1)}\left(z ;\left[t_{0}, t_{0}+k T\right]\right)>j, \forall|z|=R_{0}, \text {, } 5 \text {. } \\
& \text { or respectively, } \\
& \qquad \operatorname{Rot}_{(5.1)}\left(z ;\left[t_{0}, t_{0}+k T\right]\right)>j, \forall|z|=r_{0} \text { and } \operatorname{Rot}_{(5.1)}\left(z ;\left[t_{0}, t_{0}+k T\right]\right)<j, \forall|z|=R_{0} .
\end{aligned}
$$


Then there exist at least two initial points $z_{1} \neq z_{2}$, with $r_{0}<\left|z_{1}\right|,\left|z_{2}\right|<R_{0}$, such that the solutions $\zeta\left(\cdot ; t_{0}, z_{1}\right)$ and $\zeta\left(\cdot ; t_{0}, z_{2}\right)$ are $k T$-periodic and

$$
\operatorname{Rot}_{(5.1)}\left(z_{1} ;\left[t_{0}, t_{0}+k T\right]\right)=\operatorname{Rot}_{(5.1)}\left(z_{2} ;\left[t_{0}, t_{0}+k T\right]\right)=j .
$$

In the special case of the second order equation

$$
x^{\prime \prime}+w(t, x)=0,
$$

with $w(t, 0) \equiv 0$, which can be written as a planar Hamiltonian system like (5.1) for $\mathcal{H}(t, x, y)=\frac{1}{2} y^{2}+\int w(t, \xi) d \xi$, the condition (5.4) corresponds to the fact that the solutions $x^{(i)}(\cdot):=\zeta_{1}\left(\cdot ; t_{0}, z_{i}\right)(i=1,2)$ of $(5.5)$ possess exactly $2 j$ zeros in the interval [0, $k T[$.

Theorem 5.1 follows from a theorem by W.-Y. Ding [14], based on a result by Jacobowitz [22, 23] (see also [21, 28]). It could be deduced also from other versions of the Poincaré-Birkhoff theorem due to Franks [18], Rebelo [36] and Qian-Torres [31], where independent proofs are given. The hypothesis (5.3) implies the so-called twist condition of the Poincaré-Birkhoff theorem. We have applied Theorem 5.1 in the proof of Theorem 3.3 with $k=1$, in order to obtain $T$-periodic solutions. Applications for an integer $k \geq 2$ can lead to existence and multiplicity results for subharmonic solutions.

\subsection{A result on flow-invariant sets}

The proof of one of our key lemmas for the rotations numbers (Lemma 3.2) is based on a comparison argument which is justified by a result on flow-invariant set. In order to make our work self-contained we present now some specific details on positively invariant sets for non-autonomous differential systems which are needed in Section 3. Similar results can be also found in $[1,17,41]$.

Throughout the section, $\Omega \subset \mathbb{R}^{N}$ will denote an open set and $\Xi: J_{0} \times \Omega \rightarrow \mathbb{R}^{N}$ a continuous function with $J_{0} \subset \mathbb{R}$ an interval (that will be not restrictive to assume open). We are interested in conditions ensuring the (positive) invariance of a set $\mathcal{M} \subset \Omega$, for the solutions to the differential equation

$$
\xi^{\prime}=\Xi(t, \xi)
$$

according to the following definition.

Definition 5.1 Let $\mathcal{M} \subset \Omega$. We say that $\mathcal{M}$ is positively invariant for (5.6) if, for every $\xi: \operatorname{dom}(\xi) \rightarrow \Omega$ solution to (5.6) (being $\operatorname{dom}(\xi) \subset J_{0}$ an interval) and $t_{0} \in \operatorname{dom}(\xi)$,

$$
\left.\xi\left(t_{0}\right) \in \mathcal{M} \Longrightarrow \xi(t) \in \mathcal{M}, \quad \text { for every } t \in\right] t_{0},+\infty[\cap \operatorname{dom}(\xi)
$$

In the above definition, we have denote by $\operatorname{dom}(\xi)$ any interval on which a solution $\xi$ is defined (not necessarily a maximal one). We remark that, in literature, various concepts of (positive) invariance have been considered by different authors. In some classical results (like, for instance, the Nagumo Theorem) a weaker form of invariance is considered, namely that for any initial point belonging to $\mathcal{M}$ at least one local solution exists belonging to $\mathcal{M}$ in the forward time. Our definition refers to all the possible solutions. Clearly, 
such different points of view coincide when the (forward) uniqueness of the solutions to the initial value problems is assured.

In the following, the topological notions of interior, boundary and closure of $\mathcal{M}$ will always be meant in the subspace topology.

Lemma 5.1 Let $\mathcal{M} \subset \Omega$ be closed. Assume that, for every point $p \in \partial \mathcal{M}$, there exist $\epsilon_{p}>0$ with $\mathcal{B}\left(p, \epsilon_{p}\right) \subset \Omega$ and a $C^{1}$-function $V_{p}: \mathcal{B}\left(p, \epsilon_{p}\right) \rightarrow \mathbb{R}$ such that:

(i) $\mathcal{M} \cap \mathcal{B}\left(p, \epsilon_{p}\right)=\left\{q \in \mathcal{B}\left(p, \epsilon_{p}\right) \mid V_{p}(q) \leq 0\right\}$,

(ii) for every $t \in J_{0}$, there exists a right neighborhood $I_{t}$ of $t$ such that

$$
\left\langle\nabla V_{p}(q) \mid \Xi(s, q)\right\rangle \leq 0, \quad \text { for every } s \in \mathcal{I}_{t}, q \in \mathcal{B}\left(p, \epsilon_{p}\right) \backslash \mathcal{M} .
$$

Then $\mathcal{M}$ is positively invariant for (5.6).

Proof. Assume, by contradiction, that there exist $\xi: \operatorname{dom}(\xi) \rightarrow \Omega$ solution to (5.6) and $t_{0}, t_{1} \in \operatorname{dom}(\xi)$, with $t_{0}<t_{1}$, such that $\xi\left(t_{0}\right) \in \mathcal{M}, \xi\left(t_{1}\right) \notin \mathcal{M}$. The set $J:=\left\{s \in\left[t_{0}, t_{1}\right] \mid\right.$ $\xi(s) \in \mathcal{M}\}$ is compact and nonempty; set $t^{*}=\max J<t_{1}$ and $p^{*}=\xi\left(t^{*}\right)$. Since $p^{*} \in \partial \mathcal{M}$ and $\xi(t) \notin \mathcal{M}$ for $\left.t \in] t^{*}, t_{1}\right]$, assumption (ii) implies that

$$
\left.\left.\left\langle\nabla V_{p^{*}}(\xi(t)) \mid \Xi(t, \xi(t))\right\rangle \leq 0, \quad \text { for every } t \in\right] t^{*}, t^{*}+\delta\right],
$$

being $\delta>0$ so small that $\left[t^{*}, t^{*}+\delta\right] \subset \mathcal{I}_{t^{*}}$ and $\xi(t) \in \mathcal{B}\left(p^{*}, \epsilon_{p^{*}}\right)$ for every $\left.\left.t \in\right] t^{*}, t^{*}+\delta\right]$. Hence,

$$
V_{p^{*}}\left(\xi\left(t^{*}+\delta\right)\right)-V_{p^{*}}\left(\xi\left(t^{*}\right)\right)=\int_{t^{*}}^{t^{*}+\delta}\left\langle\nabla V_{p^{*}}(\xi(t))\right| \Xi(t, \xi(t)) d t \leq 0 .
$$

On the other hand, (i) implies that $V_{p^{*}}\left(\xi\left(t^{*}\right)\right) \leq 0<V_{p^{*}}\left(\xi\left(t^{*}+\delta\right)\right)$, contradicting (5.7).

We now turn our attention to the case in which the set $\mathcal{M}$ is a sub-level set of a scalar function $V$. This situation occurs in various applications where $V$ is a Lyapunov tpe function. Accordingly, let us suppose that

$$
\mathcal{M}:=\{p \in \Omega \mid V(p) \leq 0\},
$$

for a function $V: \Omega \rightarrow \mathbb{R}$ of class $C^{1}$. Notice that, in view of the continuity of $V, \mathcal{M} \subset \Omega$ is a closed set (relatively to $\Omega$ ).

Proposition 5.1 Suppose that the uniqueness for the Cauchy problems associated with (5.6) is ensured and let $\mathcal{M} \subset \Omega$ be defined as in (5.8). Moreover, assume that:

- $\nabla V(p) \neq 0$, for every $p \in \partial \mathcal{M}$;

- for every $t \in J_{0}$ and for every $p \in \partial \mathcal{M}$,

$$
\langle\nabla V(p) \mid \Xi(t, p)\rangle \leq 0 .
$$

Then $\mathcal{M}$ is positively invariant for (5.6). 
Proof. When (5.9) is satisfied with the strict inequality, the thesis follows directly from Lemma 5.1 (even without assuming the uniqueness for the Cauchy problems). Indeed, taking, for every $p \in \partial \mathcal{M}, \epsilon_{p}>0$ small enough and $V_{p}=\left.V\right|_{B\left(p, \epsilon_{p}\right)}$, one has that $(i)$ is clearly satisfied, while (ii) follows from (5.9), using the continuity of $V$.

We now show the conclusion in the general case. Assume, by contradiction, that $\xi$ : $\operatorname{dom}(\xi) \rightarrow \Omega$ is a solution to (5.6), $t_{0}, t_{1} \in \operatorname{dom}(\xi)$ with $t_{0}<t_{1}$ and $\xi\left(t_{0}\right) \in \mathcal{M}$, while $\xi\left(t_{1}\right) \notin \mathcal{M}$. Consider the differential equation

$$
\xi^{\prime}=\Xi_{n}(t, \xi):=\Xi(t, \xi)-\frac{1}{n} \nabla V(\xi) .
$$

Since $\Xi_{n}(t, \xi) \rightarrow \Xi(t, \xi)$ uniformly on compact subsets of $J_{0} \times \Omega$, by the general theory of ODEs we know that, for every $n$ large enough, (5.10) has a (not necessarily unique) solution $\xi_{n}(t)$ defined on $\left[t_{0}, t_{1}\right]$ and such that $\xi_{n}\left(t_{0}\right)=\xi\left(t_{0}\right)$; moreover, since we are assuming that the Cauchy problems associated with (5.6) have a unique solution, $\xi_{n}(t) \rightarrow \xi(t)$ uniformly on $\left[t_{0}, t_{1}\right]$. For every $t \in J_{0}$ and for every $p \in \partial \mathcal{M}$, it holds

$$
\left\langle\nabla V(p) \mid \Xi_{n}(t, p)\right\rangle=\langle\nabla V(p) \mid \Xi(t, p)\rangle-\frac{1}{n}|\nabla V(p)|^{2}<0 .
$$

Hence, in view of the first part of the proof, $\xi_{n}\left(t_{1}\right) \in \mathcal{M}$. Passing to the limit, we get - since $\mathcal{M}$ is closed in $\Omega-\xi\left(t_{1}\right) \in \mathcal{M}$, a contradiction.

Remark 5.1 We recall that, when $\mathcal{M} \subset \Omega$ is defined as in (5.8), one has $\partial \mathcal{M} \subset V^{-1}(0)$, the equality being satisfied whenever 0 is a regular value of $V$, i.e., $\nabla V(p) \neq 0$ for every $p \in V^{-1}(0)$. Hence, the conclusion of Proposition 5.1 holds true in the particular case when 0 is a regular value of $V$ and (5.9) is fulfilled for every $t \in J_{0}$ and $p \in V^{-1}(0)$. See [1], [41, Ch. IX] for similar results in the autonomous case.

We finally give a corollary of Proposition 5.1 which will be directly applied in Section 3. Let us consider the differential equation

$$
\left\{\begin{array}{l}
\rho^{\prime}=R(t, \rho, \theta) \\
\theta^{\prime}=\Theta(t, \rho, \theta)
\end{array}\right.
$$

being $\left.R, \Theta:[0, \tau] \times \mathbb{R}_{0}^{+} \times\right] \theta_{1}, \theta_{2}\left[\rightarrow \mathbb{R}\right.$ continuous functions (with $-\infty \leq \theta_{1}<\theta_{2} \leq+\infty$ ). In this situation, we have the following.

Corollary 5.1 Suppose that the uniqueness for the Cauchy problems associated with (5.11) is ensured and let $\gamma:] \theta_{1}, \theta_{2}\left[\rightarrow \mathbb{R}\right.$ be a function of class $C^{1}$, with $\gamma(\theta)>0$ for every $\theta \in \mathbb{R}$. Assume

$$
\left.R(t, \gamma(\theta), \theta) \leq \gamma^{\prime}(\theta) \Theta(t, \gamma(\theta), \theta), \quad \text { for every } t \in[0, \tau], \theta \in\right] \theta_{1}, \theta_{2}[.
$$

Then, for every $\left.(\rho, \theta): I \rightarrow \mathbb{R}_{0}^{+} \times\right] \theta_{1}, \theta_{2}[$ solution to (5.11) (being $I \subset[0, \tau]$ an interval) and $t_{0} \in I$,

$$
\left.\rho\left(t_{0}\right) \leq \gamma\left(\theta\left(t_{0}\right)\right) \Longrightarrow \rho(t) \leq \gamma(\theta(t)), \quad \text { for every } t \in\right] t_{0},+\infty[\cap I .
$$


Proof. Set $\left.\Omega=\mathbb{R}_{0}^{+} \times\right] \theta_{1}, \theta_{2}\left[\right.$ and $V(\rho, \theta)=\rho-\gamma(\theta)$. Since $\nabla V(\rho, \theta)=\left(1,-\gamma^{\prime}(\theta)\right)$, one has that 0 is a regular value of $V$; moreover (5.12) implies (5.9). Hence, the thesis follows from Proposition 5.1, taking into account Remark 5.1.

\section{References}

[1] H. Amann, Ordinary differential equations. An introduction to nonlinear analysis, de Gruyter Studies in Mathematics, 13, Walter de Gruyter \& Co., Berlin, 1990.

[2] C. Bandle, M.A. Pozio and A. Tesei, Existence and uniqueness of solutions of nonlinear Neumann problems, Math. Z. 199 (1988), 257-278.

[3] G.D. Birkhoff, Dynamical systems with two degrees of freedom, Trans. Amer. Math. Soc. 18 (1917), 199300 .

[4] G.D. Birkhoff, An extension of Poincaré's last geometric theorem, Acta Math. 47 (1926), 297-311.

[5] A. Boscaggin, Subharmonic solutions of planar Hamiltonian systems: a rotation number approach, Adv. Nonlinear Stud. 11 (2011), 77-103.

[6] A. Boscaggin and F. Zanolin, Pairs of positive periodic solutions of second order nonlinear equations with indefinite weight, J. Differential Equations 252 (2012), 2900-2921.

[7] A. Boscaggin and F. Zanolin, Positive periodic solutions of second order nonlinear equations with indefinite weight: multiplicity results and complex dynamics, J. Differential Equations 252 (2012), 2922-2950.

[8] A. Boscaggin and F. Zanolin, Subharmonic solutions for nonlinear second order equations in presence of lower and upper solutions, to appear in Discrete Contin. Dyn. Syst.

[9] A. Capietto, M. Henrard, J. Mawhin and F. Zanolin, A continuation approach to some forced superlinear Sturm-Liouville boundary value problems, Topol. Methods Nonlinear Anal.3 (1994), 81-100.

[10] F. Dalbono and F. Zanolin, Multiplicity results for asymptotically linear equations, using the rotation number approach, Mediterr. J. Math. 4 (2007), 127-149.

[11] W. Dambrosio, Time-map techniques for some boundary value problems, Rocky Mountain J. Math. 28 (1998), 885-926.

[12] T. Ding and F. Zanolin, Periodic solutions and subharmonic solutions for a class of planar systems of Lotka-Volterra type, World Congress of Nonlinear Analysts '92, Vol. I-IV (Tampa, FL, 1992), 395-406, de Gruyter, Berlin, 1996.

[13] T. Ding and F. Zanolin, Subharmonic solutions of second order nonlinear equations: a time-map approach, Nonlinear Anal. 20 (1993), 509-532.

[14] W.-Y. Ding, Fixed points of twist mappings and periodic solutions of ordinary differential equations, (Chinese) Acta Math. Sinica 25 (1982), 227-235.

[15] L.H. Erbe, S. Hu and H. Wang, Multiple positive solutions of some boundary value problems, J. Math. Anal. Appl. 184 (1994), 640-648.

[16] C. Fabry and P. Habets, Periodic solutions of second order differential equations with superlinear asymmetric nonlinearities, Arch. Math. (Basel) 60 (1993), 266-276.

[17] M.L.C. Fernandes and F. Zanolin, Repelling conditions for boundary sets using Liapunov-like functions. I. Flow-invariance, terminal value problem and weak persistence, Rend. Sem. Mat. Univ. Padova 80 (1988), 95-116 (1989). 
[18] J. Franks, Generalizations of the Poincaré-Birkhoff theorem, Ann. of Math. (2) 128 (1988), 139-151.

[19] G.B. Gustafson and K. Schmitt, Nonzero solutions of boundary value problems for second order ordinary and delay-differential equations, J. Differential Equations 12 (1972), 129-147.

[20] J.K. Hale, Ordinary differential equations. Second edition, Robert E. Krieger Publishing Co., Inc., Huntington, N.Y., 1980.

[21] P. Hartman, On boundary value problems for superlinear second order differential equations, J. Differential Equations 26 (1977), 37-53.

[22] H. Jacobowitz, Periodic solutions of $x^{\prime \prime}+f(x, t)=0$ via the Poincaré-Birkhoff theorem, J. Differential Equations 20 (1976), 37-52.

[23] H. Jacobowitz, Corrigendum: The existence of the second fixed point: a correction to "Periodic solutions of $x^{\prime \prime}+f(x, t)=0$ via the Poincaré-Birkhoff theorem”, J. Differential Equations 25 (1977), 148-149.

[24] M.A. Krasnosel'skiǔ, The operator of translation along the trajectories of differential equations, Translations of Mathematical Monographs, Vol. 19, American Mathematical Society, Providence, R.I. 1968.

[25] V.K. Le and K. Schmitt, Minimization problems for noncoercive functionals subject to constraints, Trans. Amer. Math. Soc. 347 (1995), 4485-4513.

[26] A. Margheri, C. Rebelo and F. Zanolin, Maslov index, Poincaré-Birkhoff theorem and periodic solutions of asymptotically linear planar Hamiltonian systems, J. Differential Equations 183 (2002), 342-367.

[27] J. Mawhin and K. Schmitt, Nonlinear eigenvalue problems with the parameter near resonance, Ann. Polon. Math. 51 (1990), 241-248.

[28] J. Moser and E.J. Zehnder, Notes on dynamical systems, Courant Lecture Notes in Mathematics, 12, New York University, Courant Institute of Mathematical Sciences, New York; American Mathematical Society, Providence, RI, 2005.

[29] Z. Opial, Sur les périodes des solutions de l'équation différentielle $x^{\prime \prime}+g(x)=0$, (French) Ann. Polon. Math 10 (1961), 49-72.

[30] H.-O. Peitgen and K. Schmitt, Global analysis of two-parameter elliptic eigenvalue problems, Trans. Amer. Math. Soc. 283 (1984), 57-95.

[31] D. Qian and P.J. Torres, Periodic motions of linear impact oscillators via the successor map, SIAM J. Math. Anal. 36 (2005), 1707-1725.

[32] P.H. Rabinowitz, Nonlinear Sturm-Liouville problems for second order ordinary differential equations, Comm. Pure Appl. Math. 23 (1970), 939-961.

[33] P.H. Rabinowitz, Pairs of positive solutions of nonlinear elliptic partial differential equations, Indiana Univ. Math. J. 23 (1973/74), 173-186.

[34] P.H. Rabinowitz, A note on pairs of solutions of a nonlinear Sturm-Liouville problem, Manuscripta Math. 11 (1974), 273-282.

[35] C. Rebelo, A note on uniqueness of Cauchy problems associated to planar Hamiltonian systems, Portugal. Math. 57 (2000), 415-419.

[36] C. Rebelo, A note on the Poincaré-Birkhoff fixed point theorem and periodic solutions of planar systems, Nonlinear Anal. 29 (1997), 291-311.

[37] C. Rebelo and F. Zanolin, Multiplicity results for periodic solutions of second order ODEs with asymmetric nonlinearities, Trans. Amer. Math. Soc. 348 (1996), 2349-2389. 
[38] C. Rebelo and F. Zanolin, On the existence and multiplicity of branches of nodal solutions for a class of parameter-dependent Sturm-Liouville problems via the shooting map, Differential Integral Equations 13 (2000), 1473-1502.

[39] R. Schaaf, Global solution branches of two-point boundary value problems, Lecture Notes in Mathematics, 1458, Springer-Verlag, Berlin, 1990.

[40] K. Schmitt, Fixed point and coincidence theorems with applications to nonlinear differential and integral equations, Séminaires de Mathématique Pure et Mécanique, Rapport 97, Université catholique de Louvain, 1976.

[41] K. Schmitt and R.C. Thompson, Nonlinear analysis and differential equations. An introduction, University of Utah, Salt Lake City, 2004.

[42] K. Schmitt and Z.-Q. Wang, On critical points for noncoercive functionals and subharmonic solutions of some Hamiltonian systems, Proceedings of the Conference on Nonlinear Differential Equations (Coral Gables, FL, 1999), 237-245, Electron. J. Differ. Equ. Conf. 5, 2000.

[43] C. Zanini, Rotation numbers, eigenvalues, and the Poincaré-Birkhoff theorem, J. Math. Anal. Appl. 279 (2003), 290-307. 